\title{
First detection of a THz water maser in NGC 7538-IRS1 with SOFIA and new 22 GHz e-MERLIN maps ${ }^{\star}$
}

\author{
F. Herpin ${ }^{1}$, A. Baudry ${ }^{1}$, A. M. S. Richards ${ }^{2}$, M. D. Gray ${ }^{2}$, N. Schneider ${ }^{3}$, K. M. Menten ${ }^{4}$, F. Wyrowski ${ }^{4}$, \\ S. Bontemps ${ }^{1}$, R. Simon ${ }^{3}$, and H. Wiesemeyer ${ }^{4}$ \\ ${ }^{1}$ Laboratoire d'astrophysique de Bordeaux, Univ. Bordeaux, CNRS, B18N, allée Geoffroy Saint-Hilaire, 33615 Pessac, France \\ e-mail: fabrice.herpin@u-bordeaux.fr \\ 2 JBCA, School of Physics and Astronomy, Univ. of Manchester, Oxford Road, M13 9PL, UK, \\ 3 I. Physik. Institut, University of Cologne, Zülpicher Str. 77, 50937 Cologne, Germany \\ ${ }^{4}$ Max-Planck-Institut für Radioastronomie, Auf dem Hügel 69, 53121 Bonn, Germany \\ Received 27 April 2017 / Accepted 20 June 2017
}

\begin{abstract}
Context. The formation of massive stars $\left(M>10 M_{\odot}, L>10^{3} L_{\odot}\right)$ is still not well understood. Accumulating a large amount of mass infalling within a single entity in spite of radiation pressure is possible if, in addition to several other conditions, enough thermal energy is released. Despite numerous water line observations over a broad range of energies obtained with the Herschel Space Observatory, observations were not able to trace the emission from the hot core around the newly forming protostellar object in most of the sources.

Aims. We wish to probe the physical conditions and water abundance in the inner layers of the host protostellar object NGC 7538IRS1 using a highly excited $\mathrm{H}_{2} \mathrm{O}$ line. Water maser models predict that several $\mathrm{THz}$ water masers should be detectable in these objects. We therefore aim to detect the $\mathrm{o}-\mathrm{H}_{2} \mathrm{O} 8_{2,7}-7_{3,4}$ line in a star forming region for the first time. Model calculations have predicted this line to show maser action.

Methods. We present SOFIA observations of the o- $\mathrm{H}_{2} \mathrm{O} 8_{2,7}-7_{3,4}$ line at $1296.41106 \mathrm{GHz}$ and a $6_{16}-5_{23} 22 \mathrm{GHz}$ e-MERLIN map of the region (the very first $22 \mathrm{GHz}$ images made after the e-MERLIN upgrade). In order to be able to constrain the nature of the emission - thermal or maser - we used near-simultaneous observations of the $22 \mathrm{GHz}$ water maser performed with the Effelsberg radiotelescope and e-MERLIN. A thermal water model using the RATRAN radiative transfer code is presented based on HIFI pointed observations. Molecular water abundances are derived for the hot core.

Results. The o- $\mathrm{H}_{2} \mathrm{O}_{2,7}-7_{3,4}$ line is detected toward NGC 7538-IRS1 with one feature at the source velocity $\left(-57.7 \mathrm{~km} \mathrm{~s}^{-1}\right)$ and another one at $-48.4 \mathrm{~km} \mathrm{~s}^{-1}$. We propose that the emission at the source velocity is consistent with thermal excitation and is excited in the innermost part of the IRS1a, in the closest circumstellar environment of the massive protostellar object. The other emission is very likely the first detection of a water THz maser line, pumped by shocks due to the IRS1b outflow, in a star-forming region. Assuming thermal excitation of the THz line, the water abundance in the hot core of NGC 7538-IRS1 is estimated to be $5.2 \times 10^{-5}$ with respect to $\mathrm{H}_{2}$.
\end{abstract}

Key words. stars: formation - masers - stars: individual: NGC 7538-IRS1 - ISM: molecules - stars: protostars - ISM: abundances

\section{Introduction}

Massive stars, despite their rarity, are important constituents of galaxies and produce most of their luminosity. The complex and still not clearly defined evolutionary sequence ranges from massive prestellar cores, high-mass protostellar objects (HMPOs), hot molecular cores, and finally to the more evolved ultra compact HII (UCHII) region stage, where the central object begins to ionize the surrounding gas (e.g., Beuther et al. 2007; König et al. 2017). The classification adopted above is of course not unique. The molecular material in star-forming regions has a wide range of temperatures and number densities (10-2000 K and $10^{4}-10^{9} \mathrm{~cm}^{-3}$ ), with different chemical characteristics. Water is a key molecule for determining the physical and chemical structure of star-forming regions because of the large abundance variations between warm and cold regions. In cool molecular clouds,

\footnotetext{
* The reduced spectra and maps (FITS files) are only available at the CDS via anonymous ftp to cdsarc.u-strasbg. fr (130.79.128.5) or via

http://cdsarc.u-strasbg.fr/viz-bin/qcat?J/A+A/606/A52
}

water is mostly found as ice on dust grains, but at temperatures $T>100 \mathrm{~K}$, the ice evaporates, increasing the gas-phase water abundance by several orders of magnitude (Fraser et al. 2001; Aikawa et al. 2008). At temperatures above $\sim 250 \mathrm{~K}$, all gasphase free oxygen may be driven into $\mathrm{H}_{2} \mathrm{O}$, increasing its abundance to $\sim 3 \times 10^{-4}$ (van Dishoeck et al. 2011).

Major progress in our understanding of interstellar water and star formation has been made with the Herschel Space Observatory. The guaranteed-time key program WISH (Water In Star-forming regions with Herschel, van Dishoeck et al. 2011) probed massive star-forming regions using water observations. To collapse, the gas must be able to release enough thermal energy, in addition to several other condistions; one main WISH goal was to determine how much of the cooling of the warm regions $(T>100 \mathrm{~K})$ is due to $\mathrm{H}_{2} \mathrm{O}$. In particular, the dynamics of the central regions has been characterized using the water lines, and the amount of cooling provided has been measured. Herschel observations of many water lines made with the highspectral resolution Heterodyne Instrument for the Far Infrared (HIFI, de Graauw et al. 2010) allowed observers to perform 
some type of tomography of the whole protostellar environment, and hence to probe the physical conditions and estimate the water abundance from a few $100 \mathrm{AU}$ to a few 10000 AU from the star (see Herpin et al. 2016). Nevertheless, observational evidence for the water abundance jump close to the forming stars that heats up their environs is still scarce (e.g., van der Tak et al. 2006; Chavarría et al. 2010; Herpin et al. 2012, 2016). While the outer abundance for the HMPOs studied in the WISH program is well constrained and estimated to be a few $10^{-8}$ in all sources, the inner abundance varies from source to source between 0.2 and $14 \times 10^{-5}$ (Herpin et al. 2016). Observing water lines involving high enough energy levels will allow us to probe the physical conditions and water abundance in the inner layers of the protostellar environment and thus to address this problem. One main difficulty is that up to now it has been impossible to (i) spatially resolve the region where the water jump occurs and (ii) to probe this region with the help of high-excitation optically thin lines.

Since the end of the Herschel mission, only the Stratospheric Observatory for Infrared Astronomy (SOFIA, Young et al. 2012) allows us to observe water in the $\mathrm{THz}$ frequency range. Interestingly, some of the $\mathrm{THz}$ water lines could even be masing. The $\mathrm{H}_{2} \mathrm{O}$ excitation model of Neufeld \& Melnick (1991) as well as the works of Yates et al. (1997) and more recently, of Daniel \& Cernicharo (2013) and Gray et al. (2016), which incorporated the most recent collisional excitation rates of water with ortho- and para- $\mathrm{H}_{2}$, all make important predictions of masing transitions in the supra- $\mathrm{THz}$ region. Several transitions could be masing that have frequencies within the L1 and L2 channels of the German Receiver for Astronomy at Terahertz Frequencies (GREAT, Heyminck et al. 2012) on board SOFIA (Heyminck et al. 2012). In particular, three predicted $\mathrm{O}-\mathrm{H}_{2} \mathrm{O}$ masing transitions and one $\mathrm{p}-\mathrm{H}_{2} \mathrm{O}$ masing transition fall in the $1.25-1.50 \mathrm{THz}$ range. Only one $\mathrm{o}-\mathrm{H}_{2} \mathrm{O}$ transition, $8_{2,7}-7_{3,4}$ at $1296.41 \mathrm{GHz}$, could be observed at the flying altitude of the SOFIA observatory (transmission $\simeq 62 \%$ ), the others are absorbed by the remaining atmosphere. Emission from this transition involves an upper energy level at $1274.2 \mathrm{~K}$ and can probe the gas deep enough into the inner layers of the hot core. We note that Neufeld et al. (2017) have just reported the first detection of the $8_{2,7}-7_{3,4}$ masing line toward an evolved star.

We have selected NGC 7538-IRS1, the best-studied massive object from our WISH program (Herpin et al. 2016; van der Tak et al. 2013), which has previously been observed in the $22 \mathrm{GHz}$ maser line and exhibits strong thermal water lines. NGC 7538-IRS1 is a relatively nearby $(2.65 \mathrm{kpc}$, Moscadelli et al. 2009) UCHII object in the complex massive star-forming region NGC 7538 surrounded by a molecular hot core. The $22 \mathrm{GHz} \mathrm{H}_{2} \mathrm{O}$ masers associated with it exhibit a complex spatial distribution, and the strongest and main concentration of the maser features (see Kameya et al. 1990; Surcis et al. 2011) is found in the direction of IRS1. Until recently (see Beuther et al. 2013), the central source was thought to be an O6 $\operatorname{star}\left(30 M_{\odot}, L \simeq 8 \times 10^{4} L_{\odot}\right)$ forming one single high-mass young stellar object (YSO). New interferometric observations of the methanol masers (Moscadelli \& Goddi 2014) have demonstrated that NGC 7538-IRS1 consists of three individual highmass YSOs named IRS1a, IRS1b, and IRS1c within 1600 AU. We note that IRS1a, b, and c are associated with the methanol maser clusters labeled by Minier et al. (2000) B+C, A, and E, respectively. The most massive YSO is IRS1a, with $\sim 25 M_{\odot}$ and a quasi-Keplerian disk of $\sim 1 M_{\odot}$ that dominates the bolometric luminosity of the region. Another massive $\left(\leq 16 M_{\odot}\right)$ and thick disk orbits the less massive (a few $M_{\odot}$ ) IRS1b object. The third source, IRS1c, is likely to be a massive YSO as well.
Several bipolar outflows or jets emanating from the IRS1 region in multiple directions have been identified and characterized. A north-south free-free ionized jet with an opening angle $\leq 30^{\circ}$ has been observed by Sandell et al. (2009), as well as a strong accretion flow toward IRS1 $(\dot{M} \sim 2 \times$ $\left.10^{-3} M_{\odot} / \mathrm{yr}\right)$. Several studies have characterized the NW-SE (PA $=-50^{\circ}$, Qiu et al. 2011) CO outflow now thought to originate from IRS1a (Moscadelli \& Goddi 2014). In addition, a NESW outflow with PA $=40^{\circ}$ has been observed by Beuther et al. (2013). An outflow driven by IRS1b that is collimated by its rotating disk has also been observed (Moscadelli \& Goddi 2014). The presence of jets or outflows and strong accretion flows makes this shocked region an ideal place for exciting masers.

In this paper we report an observational study of the NGC 7538-IRS1 region. The o- $\mathrm{H}_{2} \mathrm{O} 8_{2,7}-7_{3,4}$ emission was observed with the GREAT instrument on board SOFIA. In addition, the $22 \mathrm{GHz}$ water maser emission was observed using the Effelsberg telescope and the e-MERLIN interferometer. In Sect. 2 we describe the observations, and we present the observational data in Sect. 3. In Sect. 4 we discuss the nature of the detected $\mathrm{THz}$ water emission. In Sect. 5 we discuss the region kinematics and physical conditions, and in Sect. 6 we summarize the results of our study.

\section{Observations and data reduction}

\subsection{SOFIA observations}

Observations in single-point chopping mode of the $8_{2,7}-7_{3,4}$ line of ortho- $\mathrm{H}_{2}^{16} \mathrm{O}$ were carried out toward NGC 7538-IRS1 $\left(\alpha_{\mathrm{J} 2000}=23^{\mathrm{h}} 13^{\mathrm{m}} 45.3^{\mathrm{s}}, \delta_{\mathrm{J} 2000}=+61^{\circ} 28^{\prime} 10.0^{\prime \prime}\right)$ as part of the SOFIA Cycle 3 project on 2015 December 9, using GREAT. At the systemic velocity of NGC 7538-IRS1, the system temperature was typically $2000 \mathrm{~K}$ (SSB) and signal-band zenith opacity 0.08 . The on-source integration time was $20 \mathrm{~min}$. The chop throw was $100^{\prime \prime}$ to either side of the on-source position (chop-nod method). One channel (L1) of GREAT was tuned to the $1296.41106 \mathrm{GHz}$ water line frequency (lower sideband LSB), the other channel, the Low Frequency Array (LFA) sevenpixel array, was tuned to the [CII] $158 \mu \mathrm{m}$ line. The [CII] line was detected but a discussion of these results will be presented elsewhere. We employed the digital 4GFFT spectrometer (Klein et al. 2012) that analyzed a bandwidth of $1.5 \mathrm{GHz}$ with a spectral resolution of $0.283 \mathrm{MHz}\left(0.056 \mathrm{~km} \mathrm{~s}^{-1}\right)$. Data have later been smoothed to $1.129 \mathrm{~km} \mathrm{~s}^{-1}$. The rms of the spectra is $90 \mathrm{mK}$ at this latter spectral resolution.

The data were processed with the latest version of the GREAT calibrator and converted from the $T_{A}^{*}$ scale $\left(\eta_{f}=0.97\right)$ into main-beam temperature units, $T_{\mathrm{MB}}$, by applying the mainbeam coupling efficiency $\eta_{\mathrm{mb}}=0.69$ for the L1 channel. The conversion factor $\mathrm{Jy} / \mathrm{K}$ we applied on the $T_{A}^{*}$ data is $971 \mathrm{Jy} / \mathrm{K}$. The half-power beam width is $20.6^{\prime \prime}$ at this frequency. All spectra were calibrated for the transmission in the signal band, and the continuum-level correction for double-sideband reception was applied. Further analysis was made within the CLASS ${ }^{1}$ package. The continuum level (SSB) is $T_{\mathrm{MB}}=2.0 \mathrm{~K}$ (at 1296.4 GHz).

\subsection{Effelsberg observations}

In order to constrain the maser models and because of the variability of the maser emission, nearly contemporaneous

\footnotetext{
http://wWw.iram.fr/IRAMFR/GILDAS/
} 
observations of the $6_{16}-5_{23}$ transition of ortho- $\mathrm{H}_{2} \mathrm{O}$ (rest frequency $22.23508 \mathrm{GHz}$ ) were carried out on 2015 December 11 (integration time on-source of $15 \mathrm{~min}$ ) with the MPIfR $100 \mathrm{~m}$ telescope at Effelsberg, Germany. The half-power beam width was $\simeq 39^{\prime \prime}$ and the pointing accuracy was, on average, $4^{\prime \prime}$. We used the $1.3 \mathrm{~cm}$ double-beam secondary focus receiver (K/Jy conversion factor of 1.6) with the XFFT backend in highresolution mode (32768 channels with a spectral resolution of $\sim 0.04 \mathrm{~km} \mathrm{~s}^{-1}$ ). Data were smoothed to $0.1 \mathrm{~km} \mathrm{~s}^{-1}$ and the rms noise at this resolution is $37 \mathrm{mJy}$. The system temperature was $190 \mathrm{~K}$. All data were calibrated in Jy; the calibration parameters were derived by continuum observations of suitable flux density calibrators. The main-beam efficiency is 0.64 . The calibration uncertainty is about $10-15 \%$.

\section{3. e-MERLIN observations}

Interferometric observations of the same source of the ortho$\mathrm{H}_{2} \mathrm{O} \quad 6_{16}-5_{23}$ line emission were performed with e-MERLIN (commissioning observations) on 2016 April 20. These were the very first $22 \mathrm{GHz}$ images made after the e-MERLIN upgrade. Four telescopes were used, the longest baseline being $217 \mathrm{~km}$, with the $25 \mathrm{~m}$ Mark 2, Darnhall, and Pickmere telescopes and the $32 \mathrm{~m}$ Cambridge telescope. At the assumed distance of $2.7 \mathrm{kpc}$, the synthesized beam of 20 mas corresponds to a spatial resolution element of approximately $54 \mathrm{AU}$, but the positions of individual bright maser components can be fit with a relative accuracy $\sim 1$ AU.

The observations were made in full Stokes parameters (although only the parallel bands were processed, giving total intensity images). One spectral window (spw) of $4 \mathrm{MHz}$ was centered on the $22.23508 \mathrm{GHz}$ maser line (adjusted for the source velocity immediately before observations), divided into 512 channels, giving a spectral resolution of $0.105 \mathrm{~km} \mathrm{~s}^{-1}$ and a total useful span $\sim 50 \mathrm{~km} \mathrm{~s}^{-1}$. Two similar spw were placed either side (but they did not contain any emission). A single $125 \mathrm{MHz}$ spectral window (also with 512 channels) was placed to overlap them for calibration. The pointing position of $\alpha_{\mathrm{J} 2000}=23^{\mathrm{h}} 14^{\mathrm{m}} 01.749^{\mathrm{s}}, \delta_{\mathrm{J} 2000}=+61^{\circ} 27^{\prime} 19.80^{\prime \prime}$ was used for NGC 7538, which was approximately observed $10 \mathrm{~h}$ on target, in 6-min scans interleaved with the phase reference source $\mathbf{J} 2302+6405$. The quasar 3C 84 was used to provide the bandpass correction and flux scale, with a flux density in 2016 April of $37 \mathrm{Jy}$ (measurements kindly provided by Anne Lähteenmäki, Metsahovi, priv. comm.). The local standard of rest (LSR) correction was applied to the line data before self-calibration and imaging.

The calibration was performed in CASA, and final imaging and component fitting in AIPS. The position of the brightest maser was established as a reference position before selfcalibration. Test image cubes were made at coarse resolution to look for masers within $\sim 1.5$ arcmin radius of the pointing center (within the half-power point of the primary beam). Four fields were imaged at full resolution, covering all the emission we identified, see Table 1.

The e-MERLIN beam at $22 \mathrm{GHz}$ is not yet fully characterized. We made images without primary beam correction, and then fit 2D Gaussian components to each patch of emission above $4 \sigma_{\text {rms }}$ (rejecting components that were isolated in fewer than three channels or any obvious sidelobes of brighter components). We estimated the primary beam corrections using a scaled VLA beam, and divided the measured fluxes by the factors given in Table 1, with uncertainties increasing with distance from the pointing center. The most distant fields were also slightly affected $(<10 \%)$ by time smearing.

The noise $\sigma_{\text {rms }}$ is $\sim 20 \mathrm{mJy}$ in quiet channels for fields close to the pointing center (IRS 1-3), rising to twice that in the most distant fields. The sparse visibility plane coverage means that the brighter channels are severely dynamic-range limited, with $\sigma_{\text {rms }}$ typically $10 \%$ of peaks, or even more in more remote fields. The astrometric position accuracy is a few tens of milliarcseconds (mas); the relative accuracy depends on the signal to noise ratio and is $<1$ mas for all but the faintest or most remote components.

\subsection{HIFI observations}

The source NGC 7538-IRS1 was part of the WISH GT-KP sample. Fifteen water lines (see Table A.1) were observed with the HIFI spectrometer on board the Herschel Space Observatory in the pointed (or mapping) mode at frequencies of between 547 and $1670 \mathrm{GHz}$ in 2010 and 2011 (the observation identification numbers, obsids, are listed in Table A.1) toward NGC 7538IRS1 (same coordinates as for SOFIA observations). For the pointed observations, the double-beam switch observing mode with a throw of $3^{\prime}$ was used. The off positions were inspected and do not show any emission. The frequencies, energy of the upper levels, system temperatures, integration times, and rms noise levels at a given spectral resolution for each of the lines are provided in Table A.1.

Data were taken simultaneously in $H$ and $V$ polarizations using both the acousto-optical Wide-Band Spectrometer (WBS) with 1.1 MHz resolution and the digital autocorrelator or High-Resolution Spectrometer (HRS). Calibration of the raw data into the $T_{A}$ scale was performed by the in-orbit system (Roelfsema et al. 2012). Conversion into $T_{\mathrm{mb}}$ was made using the beam efficiency ${ }^{2}$ given in Table A.1 and a forward efficiency of 0.96 . The flux scale accuracy is estimated to be between $10 \%$ for bands 1 and 2, 15\% for bands 3 and 4, and $20 \%$ in bands 6 and $7^{1}$. Data calibration was performed in the Herschel Interactive Processing Environment (HIPE, Ott 2010) version 14. Further analysis was made within CLASS. These lines are not expected to be polarized. Thus, after inspection, data from the two polarizations were averaged together. Because HIFI is operating in double-sideband mode, the measured continuum level was divided by a factor of 2 in the figures and tables (this is justified because the sideband gain ratio is close to 1 ). We note that the p- $\mathrm{H}_{2} \mathrm{O} 5_{24}-4_{31}$ line is affected by strong baseline and ripple problems.

\section{Analysis}

\section{1. $\mathrm{THz}$ water emission}

The ortho- $\mathrm{H}_{2}^{16} \mathrm{O} \quad 8_{2,7}-7_{3,4}$ line emission is detected toward NGC 7538-IRS1 with SOFIA. As shown in Fig. 1, the line profile exhibits two features at $-57.7 \pm 0.6$ and $-48.4 \pm$ $0.5 \mathrm{~km} \mathrm{~s}^{-1}$ where $22 \mathrm{GHz}$ emission is also observed (see Fig. 2). From Gaussian fitting, the $\mathrm{S} / \mathrm{N}$ ratio of the integrated lines at -57.7 and $-48.4 \mathrm{~km} \mathrm{~s}^{-1}$ is 5.5 and 3.2 , respectively. Hereafter the -57.7 and -48.4 components are called features (1) and (2), respectively. At the frequency of the observed emissions, there is no contamination by other molecular species according to Splatalogue catalog ${ }^{3}$. The peak intensities and line widths are

\footnotetext{
2 http://herschel.esac.esa.int/twiki/pub/Sandbox/ TestHifiInfoPage/

3 http://www.cv.nrao.edu/php/splat/
} 
Table 1. e-MERLIN $22 \mathrm{GHz}$ imaging parameters.

\begin{tabular}{llrrr}
\hline \hline \multirow{2}{*}{ Field } & RA, Dec (J2000) & $\begin{array}{r}\text { Size } \\
\text { hh:mm:ss dd:mm:ss }\end{array}$ & $\begin{array}{r}\text { Flux error } \\
\%\end{array}$ & $\begin{array}{r}\text { PB correction } \\
\text { divisor }\end{array}$ \\
\hline New & $23: 13: 43.69442+61: 27: 49.3597$ & 6 & 6 & 0.95 \\
IRS11 & $23: 13: 44.98183+61: 26: 49.6800$ & 6 & 10 & 0.88 \\
IRS1-3 & $23: 13: 45.14200+61: 28: 11.4100$ & 12 & 5 & 1.0 \\
IRS9 & $23: 14: 01.75100+61: 27: 19.8000$ & 6 & 15 & 0.8 \\
\hline
\end{tabular}

Notes. The table includes: center of the square field, length of the side, fractional error in flux scale, and primary beam (PB) correction.

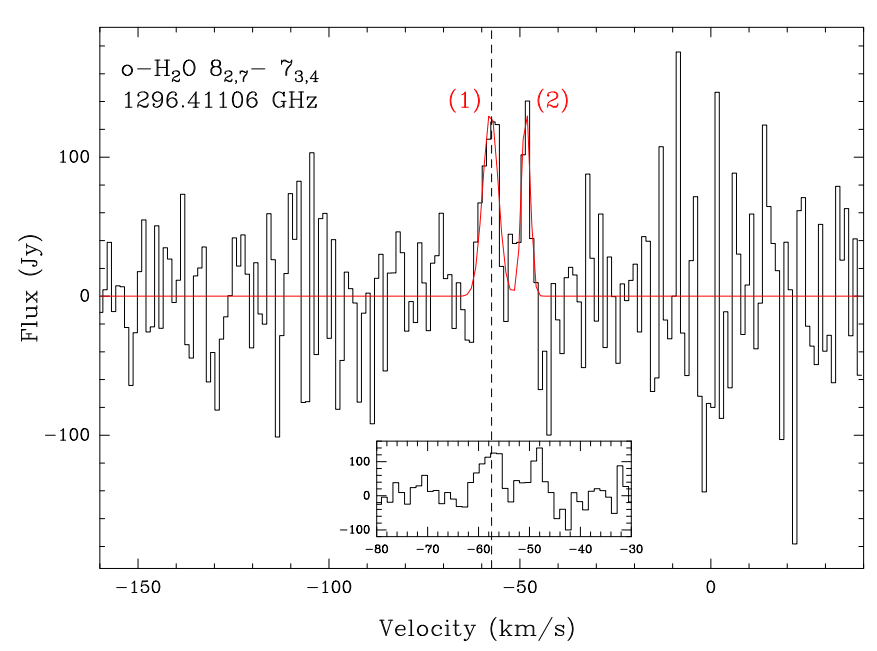

Fig. 1. Continuum-subtracted SOFIA spectra of the $0-\mathrm{H}_{2} \mathrm{O} 8_{2,7}-7_{3,4}$ line at $1296.41106 \mathrm{GHz}$ toward NGC 7538-IRS1. The vertical dotted line indicates $V_{\mathrm{LSR}}$ at $-57.4 \mathrm{~km} \mathrm{~s}^{-1}$. The spectral resolution is $1.129 \mathrm{~km} \mathrm{~s}^{-1}$. The Gaussian fit is shown in red. The small insert shows the same spectra on a zoomed velocity scale.

133.1 $\mathrm{Jy}$ and $4.7( \pm 1.3) \mathrm{km} \mathrm{s}^{-1}$ for feature (1), and 139.4 Jy and $2.6( \pm 1.3) \mathrm{km} \mathrm{s}^{-1}$ for feature (2).

The velocity and line width of feature (1) are comparable to what has been observed from the CS thermal lines $\left(v_{\mathrm{LSR}}=\right.$ $-57.4 \mathrm{~km} \mathrm{~s}^{-1}, \delta v=5.5 \pm 1 \mathrm{~km} \mathrm{~s}^{-1}$, van der Tak et al. 2000) or $\mathrm{OH}$ with SOFIA (Csengeri et al. 2012). Conversely, feature (2) is centered at a velocity different from the generally adopted source velocity. In addition, the line is narrower by a factor of 2 . The nature of these water components is discussed in Sect. 4.

\section{2. $22 \mathrm{GHz}$ single-dish observation}

Several velocity components of the $22 \mathrm{GHz}$ water maser line are seen in the Effelsberg spectra (see Fig. 2). All lines are narrow with a width on the order of $1 \mathrm{~km} \mathrm{~s}^{-1}$ or less. Two main groups of lines can be distinguished: one made of three strong lines close to the source velocity $\left.\left(\sim-(56-60) \mathrm{km} \mathrm{s}^{-1}\right)\right)$, and another group with two lines centered at -45.1 and $-48 \mathrm{~km} \mathrm{~s}^{-1}$. These lines are highly variable with time (on a timescale of several months), as shown by Felli et al. (2007) from their long-term monitoring observations. Nevertheless, most of the time, the $\sim-60 \mathrm{~km} \mathrm{~s}^{-1}$ feature is the strongest maser line.

According to the existing $22 \mathrm{GHz}$ maser maps and our eMERLIN map (see next section), all of these maser components are associated with IRS1-3. The non-detections of more distant masers are explained by the Effelsberg telescope's primary beam, which is smaller by one-third than that that of e-MERLIN.

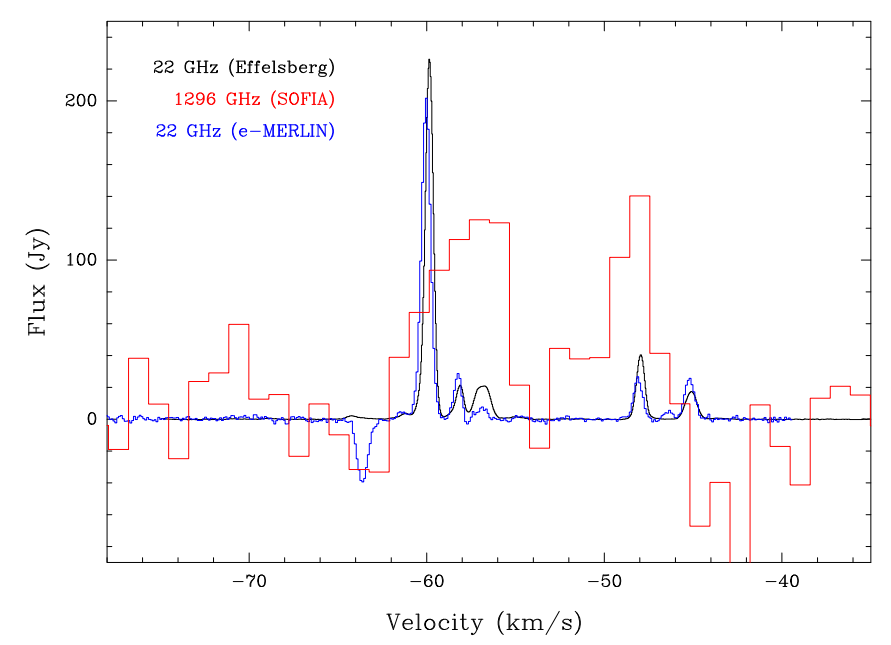

Fig. 2. Effelsberg (in black, spectral resolution $\delta \mathrm{v}$ of $0.1 \mathrm{~km} \mathrm{~s}^{-1}$ ) and e-Merlin (in blue, $\delta v=0.1 \mathrm{~km} \mathrm{~s}^{-1}$, the apparent absorption near $63.5 \mathrm{~km} \mathrm{~s}^{-1}$ is attributed to e-MERLIN residual sidelobes, see Sect. 3.3) spectra of the $6_{16}-5_{23}$ transition of ortho- $\mathrm{H}_{2} \mathrm{O}$ at $22 \mathrm{GHz}$ toward NGC 7538-IRS1 plotted over the SOFIA $\left(\delta v=1.1 \mathrm{~km} \mathrm{~s}^{-1}\right)$ spectrum of the $\mathrm{o}-\mathrm{H}_{2} \mathrm{O} 8_{2,7}-7_{3,4}$ line at $1296.41106 \mathrm{GHz}$ (in red).

\subsection{Map of the $22 \mathrm{GHz}$ maser emission}

The entire e-MERLIN map, shown in Fig. 3, encompasses the highly luminous infrared sources IRS1-3, IRS9, and IRS11 which were first identified by Werner et al. (1979). We have identified 286 individual features that can be gathered into 109 groups based on their peak velocity and coordinates. All these groups are reported in Table B.1, where their position, peak flux density $S_{v}$, peak velocity, and velocity range are listed. We have compared our maser positions with the work of Galván-Madrid et al. (2010), taking into account the proper motions from Moscadelli \& Goddi (2014), $\mu_{\mathrm{RA}}=-2.45 \mathrm{mas} / \mathrm{yr}$ and $\mu_{\text {Dec }}=-2.45$ mas $/$ yr. Both maps are consistent. Our Figs. 3 and 4 around IRS1-3 and IRS11 show maser positions very close to those labeled $\mathrm{M}$ and $\mathrm{S}$ in Table 2 of Galván-Madrid et al. (2010) and are shown here as green crosses. Moreover, the total size of the image around IRS1-3 (12", a size comparable to the SOFIA beam at $1.3 \mathrm{THz}$ ) was used to synthesize the e-MERLIN $22 \mathrm{GHz}$ spectrum, which we compare in Fig. 2 with the line profile obtained at Effelsberg in order to verify the flux density scale (e-MERLIN data are commissioning data), and to check that the interferometer is detecting the entire flux. Both line profiles are very similar, except for the apparently negative feature in the 63.5 to $-64 \mathrm{~km} \mathrm{~s}^{-1}$ velocity range, which is due to residual sidelobes arising from strong IRS11 emission in this velocity range. The maser variability over this short time-period (four months) is thus not significant and the entire flux is detected. 

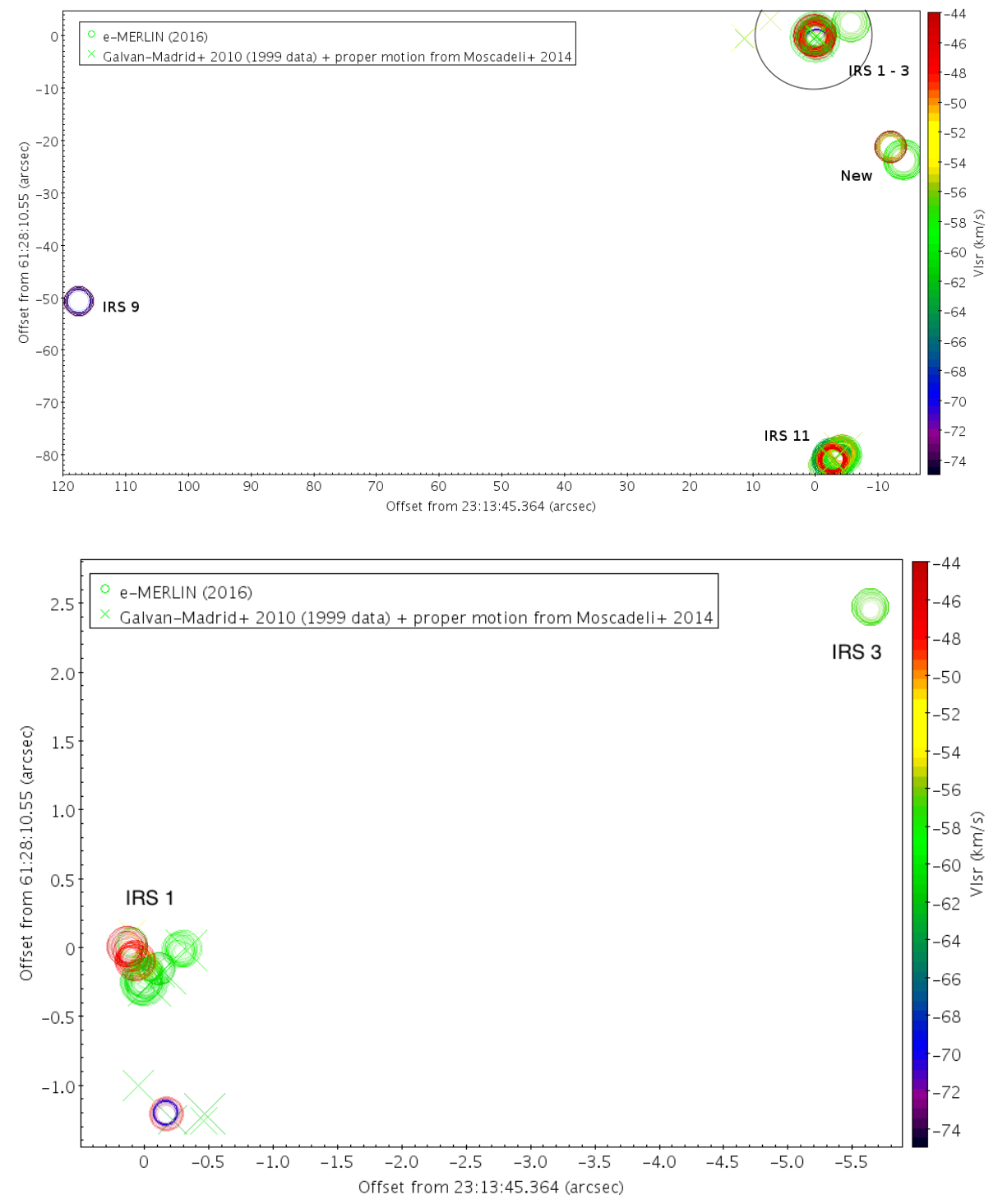

Fig. 3. Entire e-MERLIN map of $22 \mathrm{GHz}$ maser emission from NGC 7538, including IRS1-3, IRS9, and IRS11, of the $22 \mathrm{GHz}$ water maser line. Colored circles show the relative position of individual maser features, with color denoting the maser $V_{\mathrm{LSR}}$, according to the colorvelocity conversion code reported on the right side of the panel. The water maser positions reported by Galván-Madrid et al. (2010), corrected for proper motion (Moscadelli \& Goddi 2014), are shown as crosses (same colorvelocity conversion code) for comparison. The beam of the SOFIA observation is overlaid in black for comparison.

Fig. 4. As in Fig. 3, but zoomed on IRS1-3.

We detect 68 maser groups for IRS11, some of them very bright with $S_{v}$ well above $100 \mathrm{Jy}$, spanning a velocity range from -44.75 to $-65.08 \mathrm{~km} \mathrm{~s}^{-1}$. The maximum flux is observed at $-60 \mathrm{~km} \mathrm{~s}^{-1}$ (964 Jy) in IRS1. Only three main groups of masers are observed toward IRS9, and they are blueshifted ( - (69-79) $\mathrm{km} \mathrm{s}^{-1}$ ) compared to IRS1 source velocity. The strongest maser group peaks at 39 Jy in IRS9.

Approximately 25 arcsec southwest from IRS1 (offsets $\sim-(12-14)^{\prime \prime}$ and $\left.-(21-24)^{\prime \prime}\right)$, a new source has been found that exhibits powerful maser emission (up to $\sim 200 \mathrm{Jy}$ for group 5) and velocities from -45 to $-63 \mathrm{~km} \mathrm{~s}^{-1}$ (see Fig. 3 and Table B.1). The feature "E" observed by Kameya et al. (1990) with $V_{\mathrm{LSR}}=$ $-63.3 \mathrm{~km} \mathrm{~s}^{-1}$ at $\alpha_{\mathrm{J} 2000}=23^{\mathrm{h}} 13^{\mathrm{m}} 42.51^{\mathrm{s}}, \delta_{\mathrm{J} 2000}=+61^{\circ} 27^{\prime} 45.1^{\prime \prime}$, that is at offset $\sim-20^{\prime \prime}$ and $-25^{\prime \prime}$, is much farther away from this new spot.

A first zoom of the IRS1-3 region is shown in Fig. 4. The IRS3 source lies to the NW with offsets from IRS 1 of $-5.5^{\prime \prime}$ and $2.5^{\prime \prime}$ in RA-Dec, respectively. Two main groups are detected for IRS3, they are moderately bright (14-88 Jy) and span a small velocity range (from $\sim-56$ to $-58 \mathrm{~km} \mathrm{~s}^{-1}$ ). At this scale, IRS1 is made of what Surcis et al. (2011) called the north source (the main source) and the nouth source, $1.2^{\prime \prime}$ south. In addition to the maser group at $\sim-70 \mathrm{~km} \mathrm{~s}^{-1}$ for IRS1, which has also been observed by these authors, we detect maser spots at redder velocities of $\sim-46 \mathrm{~km} \mathrm{~s}^{-1}$.

The close-up view of $\mathrm{H}_{2}^{16} \mathrm{O}$ maser features around NGC 7538-IRS1 North (see Fig. 5) reveals 26 maser groups that can be split into two distinct velocity ranges: a first large group of masers within $\pm 3 \mathrm{~km} \mathrm{~s}^{-1}$ from the main source LSR velocity, represented in blue in Fig. 5, and a second group of redshifted masers whose LSR velocity is between -45 and $-48 \mathrm{~km} \mathrm{~s}^{-1}$. The "blue" group is made of numbers of features that are concentrated very close to IRS1a (as defined by Moscadelli \& Goddi 2014) plus several spots spread in the northwest. The "red" group masers lying north of IRS1a are less well aligned along a NE-SW direction. The maser spatial distribution is discussed in more detail in Sect. 4.1.

\section{Nature of the THz water emission}

\subsection{Spatial origin}

Considering the SOFIA beam size $\left(\simeq 21^{\prime \prime}\right)$ and the approximate $12^{\prime \prime}$ square size of the image made by e-MERLIN (primary beam, see Table 1) toward IRS1-3 (see Figs. 3 and 4), the detected $\mathrm{THz}$ water emission can only originate from IRS1-3. Then, comparing the velocity range of features (1) and (2) with 


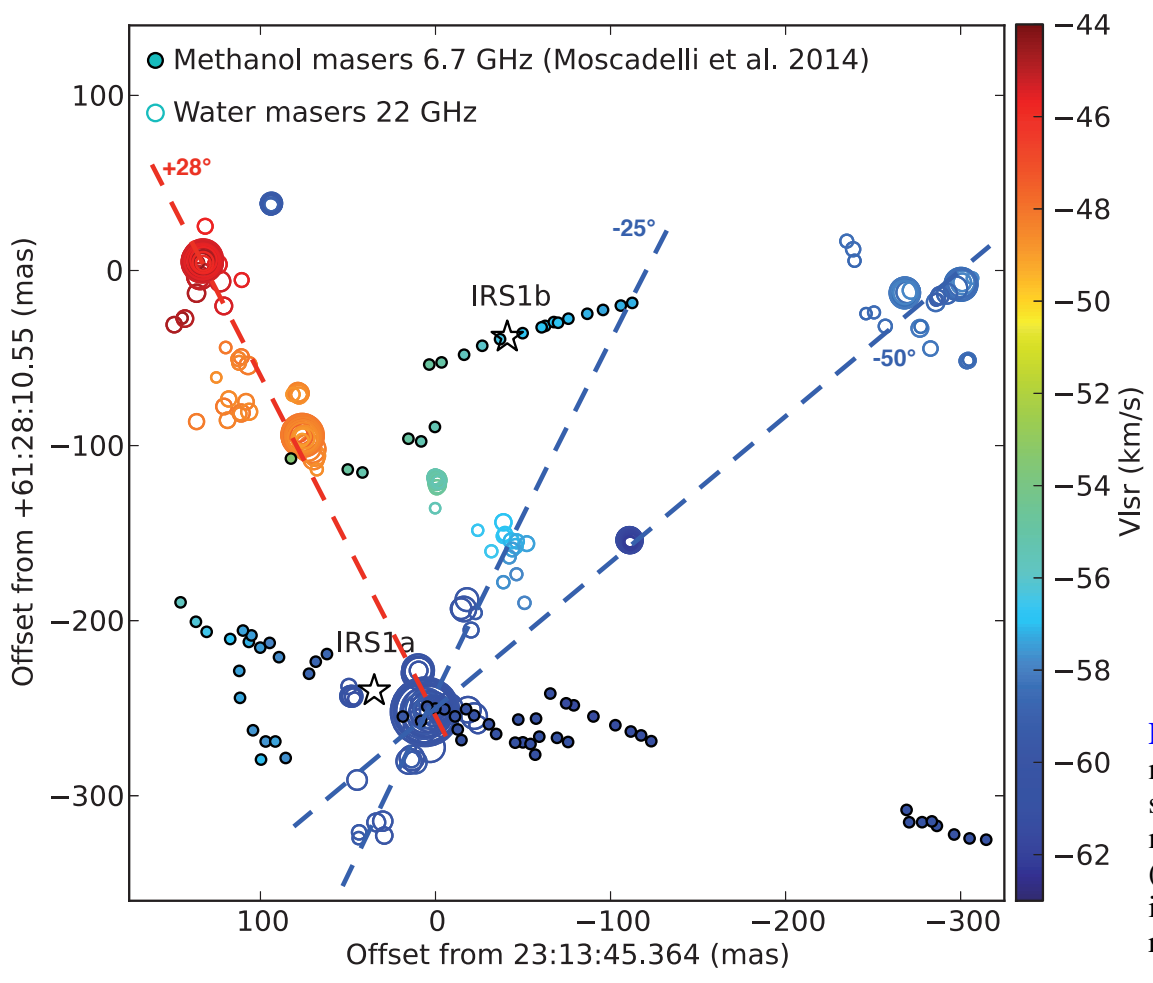

Fig. 5. Zoom toward IRS1 north of the e-MERLIN map shown in Fig. 3. Colored empty and filled circles show the relative position of individual $22 \mathrm{GHz}$ water masers (this paper) and $6.7 \mathrm{GHz}$ methanol maser features (Moscadelli \& Goddi 2014), respectively. $V_{\text {LSR }}$ is according to the color-velocity conversion code shown on the right side of the panel. the velocity of maser spots detected by e-MERLIN (Figs. 2 and 4 and Table B.1), we can infer the following:

- water in IRS1 North or IRS3 can give rise to feature (1),

- only IRS1 North exhibits $22 \mathrm{GHz}$ maser emission at velocities $\left(\sim-48.4 \mathrm{~km} \mathrm{~s}^{-1}\right)$ similar to feature (2) $\mathrm{THz}$ emission.

At the scale of Fig. 5, we can see that the sizes of the respective emitting regions for maser features around -48 and $-58 \mathrm{~km} \mathrm{~s}^{-1}$ are comparable, but not spatially coincident. Maser spots at velocities similar to $\mathrm{THz}$ feature (2) emission are located $0.2^{\prime \prime}$ northeast of the high-mass YSO IRS1a and even more than $0.1^{\prime \prime}$ east of the massive protostar IRS $1 \mathrm{~b}$. We also conclude that a similar beam dilution of the two maser features is obtained with the Effelsberg $22 \mathrm{GHz}$ beam $\left(\simeq 39^{\prime \prime}\right)$.

\subsection{Thermal or maser?}

\subsubsection{Thermal modeling}

All water lines observed toward NGC 7538-IRS1 with HIFI are shown in Fig. 6. A detailed analysis of three low- $J \mathrm{H}_{2}^{16} \mathrm{O}$ line profiles has been made by van der Tak et al. (2013) in several HMPOs, including NGC 7538-IRS1. Several lines from the rare isotopologue $\mathrm{H}_{2}^{18} \mathrm{O}$ and the para-ground-state line of $\mathrm{H}_{2}^{17} \mathrm{O}$ were detected in addition to the $\mathrm{H}_{2}^{16} \mathrm{O}$ lines toward NGC 7538-IRS1 (see Fig. 6, left).

A Gaussian fit to these line profiles (see Table 2) indicates, depending on the line, three velocity components: a narrow (3.2-4.6 $\left.\mathrm{km} \mathrm{s}^{-1}\right)$, a medium (5.1-9.3 $\mathrm{km} \mathrm{s}^{-1}$ ), and a broad $\left(>12.2 \mathrm{~km} \mathrm{~s}^{-1}\right.$ ) velocity component. The physical origin of these components (attributed to a dense core, an envelope, and an outflow) has been discussed in several papers (e.g., Herpin et al. 2016). All of these components in NGC 7538-IRS1 are globally centered at a velocity similar to that of the $\mathrm{THz}$ feature (1), that is, $\sim-57 \mathrm{~km} \mathrm{~s}^{-1}$. No emission is detected at $-48 \mathrm{~km} \mathrm{~s}^{-1}$, which is thus a first indication of the nonthermal origin of this emission.
Following the method of Herpin et al. (2012, 2016), we modeled all water line profiles in a single spherically symmetrical model using the $1 \mathrm{D}$ radiative transfer code RATRAN (Hogerheijde \& van der Tak 2000). The envelope temperature $(20-1130 \mathrm{~K})$ and number density $\left(1.1 \times 10^{5}-3.8 \times 10^{8} \mathrm{~cm}^{-3}\right)$ structure for the hot core is taken from van der Tak et al. (2013). This analysis assumes a single source within the HIFI beam, hence encompassing the IRS1-3 substructure. The outflow parameters, intensity and width, come from our Gaussian fit (see Table 2) for the broad component. The envelope contribution is parametrized with the water abundance (outer $\chi_{\text {out }}$ for $T<$ $100 \mathrm{~K}$, inner $\chi_{\text {in }}$ for $T>100 \mathrm{~K}$, assuming a jump in the abundance in the inner envelope at $100 \mathrm{~K}$ due to the evaporation of ice mantles), the turbulent velocity $\left(v_{\text {turb }}\right)$, and the infall velocity $\left(v_{\text {inf }}\right)$. We adopt the following standard abundance ratios for all the lines: 4.5 for $\mathrm{H}_{2}^{18} \mathrm{O} / \mathrm{H}_{2}^{17} \mathrm{O}$ (Thomas \& Fuller 2008), 614 for $\mathrm{H}_{2}^{16} \mathrm{O} / \mathrm{H}_{2}^{18} \mathrm{O}$ (based on Wilson \& Rood 1994), and 3 for ortho/para $\mathrm{H}_{2} \mathrm{O}$.

Considering that the width of the velocity components is not the same for all lines (see Table 2), a model with an equally turbulent velocity for all lines does not fit the data well. The best result (see Fig. 6) is obtained with a turbulent velocity of $1.5-2.5 \mathrm{~km} \mathrm{~s}^{-1}\left(5.5 \mathrm{~km} \mathrm{~s}^{-1}\right.$ is even needed for the doubtful p$\mathrm{H}_{2} \mathrm{O}_{24}-4_{31}$ line) for $\mathrm{H}_{2}^{16} \mathrm{O}$, depending on the modeled line, and $2.5 \mathrm{~km} \mathrm{~s}^{-1}$ for the rare isotopologues lines. No infall is needed at the scale probed by the HIFI lines. All modeled lines are centered at roughly $-57.4 \pm 0.5 \mathrm{~km} \mathrm{~s}^{-1}$.

Modeling of the entire set of observed lines constrains the water abundance, but only a few lines are optically thin enough to probe the inner part of the envelope. We hence derive $\mathrm{H}_{2}^{16} \mathrm{O}$ abundances relative to $\mathrm{H}_{2}$ of $8 \times 10^{-6}$ in the inner part and of $2 \times 10^{-8}$ in the outer part.

We then applied the results of the above thermal model to feature (1) of the $\mathrm{THz}$ o- $\mathrm{H}_{2} \mathrm{O} 8_{2,7}-7_{3,4}$ line (we do not see any thermal line corresponding to feature 2), assuming the same abundance and $v_{\text {turb }}=2.5 \mathrm{~km} \mathrm{~s}^{-1}$. Figure 7 shows that we do not reproduce the observed line, the intensity being only half 
F. Herpin et al.: First detection of a THz water maser in NGC 7538-IRS1 with SOFIA and new 22 GHz e-MERLIN maps

Table 2. Observed line emission parameters for the detected lines with HIFI toward NGC 7538-IRS1.

\begin{tabular}{|c|c|c|c|c|c|c|c|c|c|}
\hline Line & $\begin{array}{l}T_{\mathrm{mb}} \\
{[\mathrm{K}]}\end{array}$ & $\begin{array}{c}T_{\text {cont }} \\
{[\mathrm{K}]}\end{array}$ & $\begin{array}{c}v_{\text {nar }} \\
{\left[\mathrm{km} \mathrm{s}^{-1}\right]}\end{array}$ & $\begin{array}{c}\Delta v_{\mathrm{nar}} \\
{\left[\mathrm{km} \mathrm{s}^{-1}\right]}\end{array}$ & $\begin{array}{c}v_{\text {med }} \\
{\left[\mathrm{km} \mathrm{s}^{-1}\right]}\end{array}$ & $\begin{array}{c}\Delta v_{\text {med }} \\
{\left[\mathrm{km} \mathrm{s}^{-1}\right]}\end{array}$ & $\begin{array}{c}v_{\mathrm{br}} \\
{\left[\mathrm{km} \mathrm{s}^{-1}\right]}\end{array}$ & $\begin{array}{c}\Delta v_{\mathrm{br}} \\
{\left[\mathrm{km} \mathrm{s}^{-1}\right]}\end{array}$ & $\tau$ \\
\hline $\mathrm{o}-\mathrm{H}_{2}^{18} \mathrm{O} 1_{10}-1_{01}$ & 0.63 & 0.5 & & & $-58.4 \pm 0.4$ & $5.2 \pm 1.1$ & & & \\
\hline $\mathrm{p}-\mathrm{H}_{2}^{18} \mathrm{O} 2_{02}-1_{11}$ & 1.90 & 1.7 & $-59.5 \pm 0.2$ & $3.9 \pm 0.5$ & & & & & \\
\hline $\mathrm{o}-\mathrm{H}_{2}^{18} \mathrm{O} 3_{12}-3_{03}$ & 2.27 & 2.0 & & & $-58.2 \pm 0.2$ & $5.1 \pm 0.3$ & & & \\
\hline $\mathrm{p}-\mathrm{H}_{2}^{18} \mathrm{O} 1_{11}-0_{00}$ & 2.22 & 2.0 & & & $-57.7 /-64.3^{a} \pm 0.2$ & $5.1 / 8.8 \pm 0.2$ & & & 0.03 \\
\hline $\mathrm{p}-\mathrm{H}_{2}^{17} \mathrm{O} 1_{11}-0_{00}$ & 1.9 & 2.0 & $-56.7 \pm 0.3^{a}$ & $3.2 \pm 0.6$ & & & & & 0.04 \\
\hline $\mathrm{o}-\mathrm{H}_{2} \mathrm{O} 1_{10}-1_{01}$ & 5.21 & 0.5 & $-57.1^{a} /-54.7^{a} \pm 0.2$ & $2.0 / 3.9 \pm 0.2$ & & & $-57.2 \pm 0.2$ & $13.0 \pm 0.3$ & 0.9 \\
\hline $\mathrm{p}-\mathrm{H}_{2} \mathrm{O} 2_{11}-2_{02}$ & 6.86 & 0.8 & $-57.5 \pm 0.1$ & $4.3 \pm 0.1$ & & & $-57.6 \pm 0.1$ & $15.2 \pm 0.3$ & \\
\hline $\mathrm{p}-\mathrm{H}_{2} \mathrm{O} 5_{24}-4_{31}^{b}$ & 1.75 & 1.6 & & & & & $-57.6 \pm 0.7$ & $12.2 \pm 1.6$ & \\
\hline p- $\mathrm{H}_{2} \mathrm{O} 2_{02}-1_{11}$ & 7.92 & 1.6 & $-57.4 \pm 0.1$ & $4.4 \pm 0.1$ & & & $-57.7 \pm 0.1$ & $12.3 \pm 0.3$ & \\
\hline $\mathrm{o}-\mathrm{H}_{2} \mathrm{O} 3_{12}-3_{03}$ & 6.32 & 2.0 & $-57.3 \pm 0.1$ & $3.4 \pm 0.1$ & $-57.8 \pm 0.1$ & $8.5 \pm 0.2$ & & & \\
\hline $\mathrm{p}-\mathrm{H}_{2} \mathrm{O} 1_{11}-0_{00}{ }^{c}$ & 0.1 & 2.0 & $-56.1 \pm 0.4^{a}$ & $4.6 \pm 0.1$ & $-51.2 \pm 0.4^{a}$ & $9.3 \pm 0.6$ & $-55.8 \pm 0.2$ & $24.0 \pm 0.4$ & 2.9 \\
\hline $\mathrm{o}-\mathrm{H}_{2} \mathrm{O} 2_{21}-2_{12}$ & 5.9 & $4.0^{d}$ & & & $-60.6^{a} /-56.3 \pm 0.3$ & $5.1 / 8.7 \pm 0.5$ & & & 0.4 \\
\hline $\mathrm{o}-\mathrm{H}_{2} \mathrm{O} 2_{12}-1_{01}$ & 0.0 & $4.0^{d}$ & & & $-58.2^{a} /-52.1 \pm 0.3$ & $5.7 / 8.1 \pm 0.5$ & & & $>7$ \\
\hline
\end{tabular}

Notes. $T_{\mathrm{mb}}$ is the peak temperature with continuum. $T_{\text {cont }}$ is the real continuum temperature (single sideband). $v$ is the Gaussian component peak velocity. $\Delta v$ is the velocity full-width at half-maximum (FWHM) of the narrow, medium, and broad components. The opacity $\tau$ is from absorption lines. ${ }^{(a)}$ In absorption; ${ }^{(b)}$ line suffering from strong baseline and ripples problems; ${ }^{(c)}$ WBS data; ${ }^{(d)}$ the continuum level is not reliable.

what is observed. We can perfectly reproduce feature (1) with an increased water inner abundance of $5.2 \times 10^{-5}$ (see blue line in Fig. 7). We also successfully applied this new model to the $\mathrm{H}_{2}^{18} \mathrm{O} 3_{13}-2_{20}$ line $\left(E_{\text {up }} \simeq 200 \mathrm{~K}\right)$, reproducing the line intensity and width observed by van der Tak et al. (2006).

Adopting now the water inner abundance deduced from our modeling of the $\mathrm{o}-\mathrm{H}_{2} \mathrm{O} 8_{2,7}-7_{3,4}$ line, we again modeled the HIFI water thermal lines (in blue in Fig. 6). The result is less satisfactory, especially for the $\mathrm{p}-\mathrm{H}_{2} \mathrm{O} 5_{24}-4_{31}$ (but this observation is affected by line ripples, and the baseline determination is also affected by methanol line blending) and rare isotopologue lines. This might reflect the limitations of our symmetrical 1D model. It is also possible that the $8_{2,7}-7_{3,4}$ line detected by SOFIA emanates from the inner part of the hot core, while the water lines observed with HIFI are from somewhat cooler regions farther out. Thus, a temperature gradient could explain our results without requiring a higher water abundance throughout the region. On the other hand, feature (1) of the $1296 \mathrm{GHz}$ water line and the $\mathrm{H}_{2}^{18} \mathrm{O} 3_{13}-2_{20}$ line can be well matched by a thermal emission profile if the water abundance is increased. Nevertheless, nonthermal effects cannot be excluded: our RATRAN model could miss a nonthermal contribution at $1296 \mathrm{GHz}$, which, in that case, would be on the order of $50 \%$.

\subsubsection{Maser modeling}

We have made quasi-contemporaneous observations of the potentially masing $\mathrm{o}-\mathrm{H}_{2} \mathrm{O} 8_{2,7}-7_{3,4}$ transition at $1296 \mathrm{GHz}$ and of the $22 \mathrm{GHz}$ maser line to provide line intensity ratios enabling us to estimate the physical conditions leading to these maser emissions. Line intensity ratios are less dependent on the cloud geometry, and in the saturated regime, such ratios tend to be independent of the exact ratio of the beaming angles, which should then be close to one.

We estimate the 1296/22 opacity ratio from the recently published models of Gray et al. (2016). These models incorporate 411 levels and 7597 radiative transitions, the most recent collision rates, and line overlap effects. Even if these models have been developed primarily for evolved stars, the broad physical parameter space (including $T_{\text {dust }}$ rising from $50 \mathrm{~K}$ to $350 \mathrm{~K}$ ) that has been explored can be used for the conditions considered here. There is a significant overlap between conditions supporting the $22 \mathrm{GHz}$ and $1296 \mathrm{GHz}$ masers, for instance, both could come from gas above $1000 \mathrm{~K}$ with $\mathrm{o}-\mathrm{H}_{2} \mathrm{O}$ number densities between $10^{4}$ and $2 \times 10^{5} \mathrm{~cm}^{-3}$ (i.e., $n_{\mathrm{H}_{2}} \sim 3 \times 10^{9}-6 \times 10^{10} \mathrm{~cm}^{-3}$, taking $3 \times 10^{-5}$ as standard conversion factor from $n\left(\mathrm{H}_{2} \mathrm{O}\right)$ to $n\left(\mathrm{H}_{2}\right)$, Gray et al. 2016). However, strong $1296 \mathrm{GHz}$ maser emission is obtained for a relatively narrow range of physical conditions, typically $T_{K}$ around 1000 to $3000 \mathrm{~K}$ and $n_{\mathrm{H}_{2} \mathrm{O}}$ around $10^{4}-10^{5} \mathrm{~cm}^{-3}$, while $22 \mathrm{GHz}$ masers are excited within 500 to $3000 \mathrm{~K}$ and for a broader range of $\mathrm{H}_{2} \mathrm{O}$ densities up to several $10^{5} \mathrm{~cm}^{-3}$. Compared to the $22 \mathrm{GHz}$ inversion, the $1296 \mathrm{GHz}$ inversion is biased toward higher values of $T_{K}$ and it is lost with increasing dust temperatures, namely a low value of $T_{\text {dust }}$, around $50 \mathrm{~K}$, is preferred. The dust temperature is unlikely to be much higher than $50 \mathrm{~K}$ in shocked gas. Assuming $T_{\text {dust }}=50 \mathrm{~K}$, Gray et al. (2016) predicted $\tau(1296) / \tau(22) \sim 1$.9. This ratio does not result from specific conditions representing the observed source. It is, instead, the ratio of the maximum $1296 \mathrm{GHz}$ depth to the maximum found at $22 \mathrm{GHz}$ from models covering a large parameter space. Saturation is also not accounted for in this ratio. Nevertheless, the 1.9 ratio suggests that the flux density and brightness temperature at $1296 \mathrm{GHz}$ can be several times that at $22 \mathrm{GHz}$. To compare features (1) and (2) to our model predictions, we derive the line peak ratios from Fig. 8 where all spectra have been smoothed to the same spectral resolution. We derive $S(1296) / S(22)=1.2$ and 7.4 for features (1) and (2), respectively, but cannot directly estimate the brightness temperature of the $1296 \mathrm{GHz}$ emission since its spatial extent is unknown. At $-48 \mathrm{~km} \mathrm{~s}^{-1}$, feature (2) is definitely much brighter in the $1296 \mathrm{GHz}$ line than at $22 \mathrm{GHz}$. This is in agreement with maser conditions suggested from the 1.9 opacity ratio above. We further note that feature (2) is nearly 2.5 times narrower than feature (1) suggesting again that the former is not thermal. Assuming that the 1296 and $22 \mathrm{GHz}$ emissions have a similar spatial extent, we derive from the observed $\mathrm{S}(1296) / \mathrm{S}(22)=7.4$ at $-48 \mathrm{~km} \mathrm{~s}^{-1}, T_{\mathrm{b}}(1296)=T_{\mathrm{b}}(22) \times 2.2 \times 10^{-3}$ and, because the condition $T_{\mathrm{b}}(22)$ on the order of or greater than $10^{6} \mathrm{~K}$ is easily met at $22 \mathrm{GHz}$ (e.g., Elitzur et al. 1992), we get $T_{\mathrm{b}}(1296)$ greater than $2200 \mathrm{~K}$, that is, suprathermal emission. We stress 

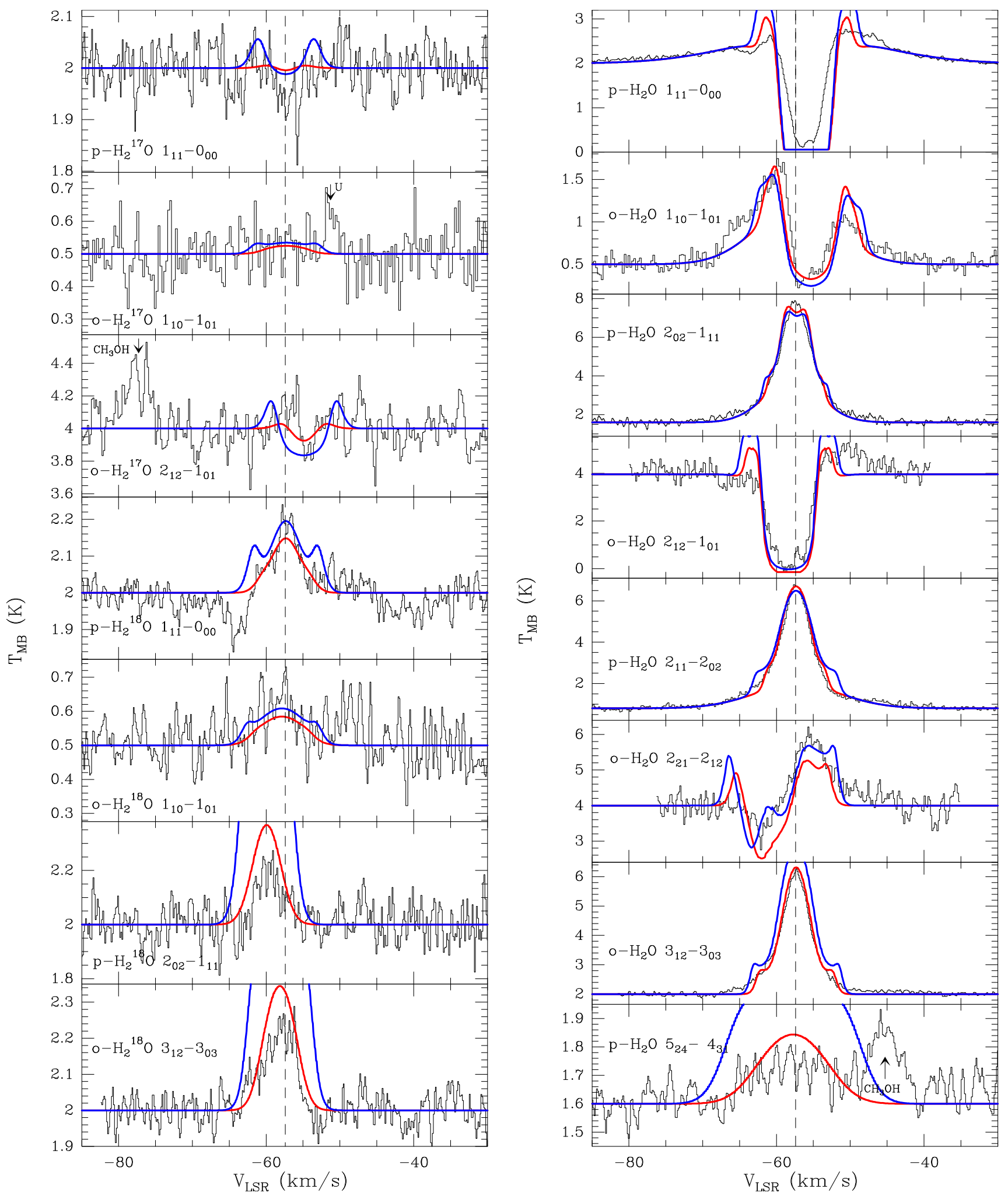

Fig. 6. HIFI spectra of $\mathrm{H}_{2}^{17} \mathrm{O} / \mathrm{H}_{2}^{18} \mathrm{O}$ (left) and $\mathrm{H}_{2}^{16} \mathrm{O}$ (right) lines (in black), with the continuum for NGC 7538-IRS1 pointed position. The best-fit model is shown as a red line over the spectra $\left(\chi_{\text {in }}\left(\mathrm{H}_{2} \mathrm{O}\right)=8 \times 10^{-6}\right.$ and $\left.\chi_{\text {out }}\left(\mathrm{H}_{2} \mathrm{O}\right)=4 \times 10^{-8}\right)$. The model adopting the SOFIA water inner abundance $\left(5.2 \times 10^{-5}\right)$ is shown as a blue line above the spectra. Vertical dotted lines indicate the $V_{\mathrm{LSR}}\left(-57.4 \mathrm{~km} \mathrm{~s}^{-1}\right.$ from the line modeling). The spectra have been smoothed to $0.2 \mathrm{~km} \mathrm{~s}^{-1}$, and the continuum is divided by a factor of two.

that deriving an exact value for $T_{\mathrm{b}}(1296)$ is impossible since we have no spatial information. When we use the e-MERLIN resolution of 20 mas as an upper limit to the beamed size of maser spots, however, we obtain $T_{\mathrm{b}}$ in the range $>10^{6}$ to $>10^{9} \mathrm{~K}$, with a mean value of $>3 \times 10^{7} \mathrm{~K}$. The beamed area of maser spots is likely to be at least two orders of magnitude smaller, giving an average $T_{\mathrm{b}}$ at least $10^{9} \mathrm{~K}$. This lower limit to $T_{\mathrm{b}}$ strongly suggests, but does not prove, the maser nature of this emission. 


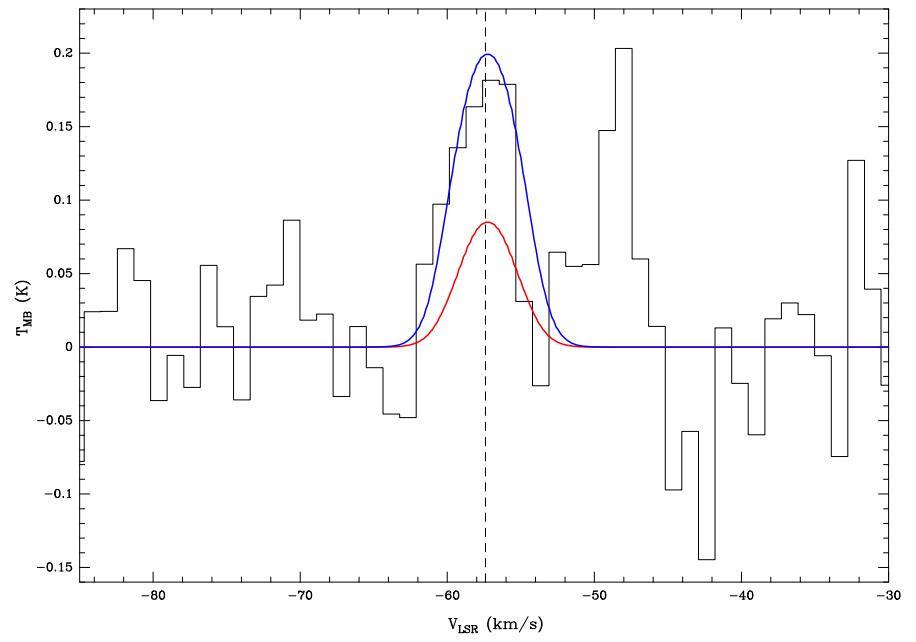

Fig. 7. SOFIA spectra of the o- $\mathrm{H}_{2} \mathrm{O} 8_{2,7}-7_{3,4}$ line showing for feature (1) the best-fit thermal model in red from our HIFI data (see Fig. 6) and a model adopting a water inner abundance of $5.2 \times 10^{-5}$ (blue profile).

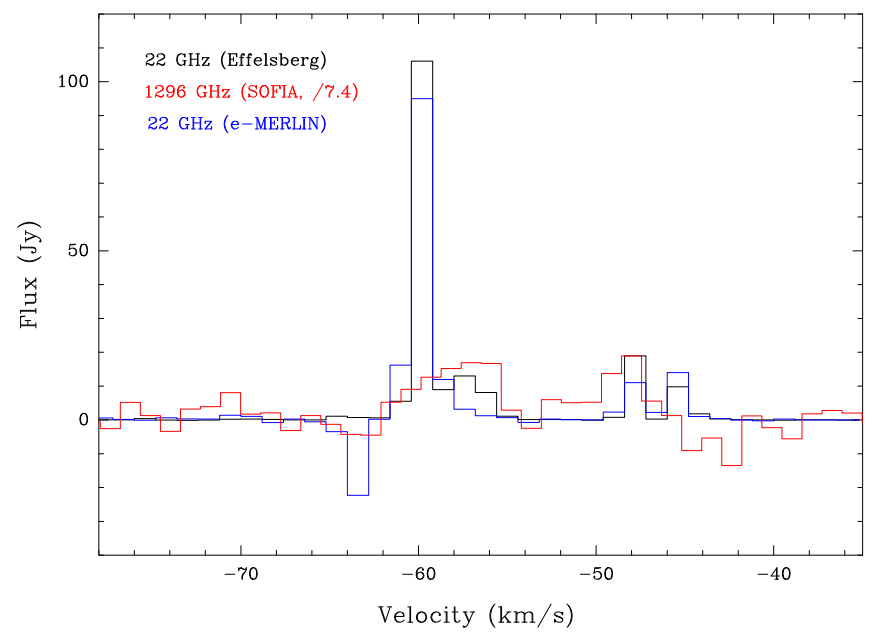

Fig. 8. As in Fig. 2, with all spectra smoothed to a spectral resolution of $1.1 \mathrm{~km} \mathrm{~s}^{-1}$. The SOFIA fluxes have been divided by a factor 7.4.

Our conclusion is thus that the feature (2) water emission is likely a maser. The absence of the typical narrow substructure in the line profile could just be due to the low $\mathrm{S} / \mathrm{N}$ (and spectral resolution) that hides it. Observations with some instrument that has much higher spatial resolution at $1296 \mathrm{GHz}$ would be the only way to unambiguously prove it is a maser.

\section{Discussion}

\subsection{Water content}

Depending on the excitation of the $\mathrm{o}-\mathrm{H}_{2} \mathrm{O} 8_{2,7}-7_{3,4}$ line, purely thermal or not, we have shown that the water inner abundance might differ by more than one order of magnitude in the hot core of NGC 7538-IRS1. From the integrated fluxes measured for the lines (considered in this study) that are at least partly in emission, we derived the water luminosities and then estimated, assuming isotropic radiation, that the minimum total HIFI water luminosity is $0.6 L_{\odot}$ (equal to the sum of all individual observed luminosities). Even though the true water emission from the inner parts might be much greater, the cool envelope absorbs much of the emission. This confirms the low contribution of water cooling to the total far-IR gas cooling compared to the cooling from other species (Karska et al. 2014; Herpin et al. 2016). Moreover, from the modeling, and assuming that the feature (1) water emission is purely thermal, we estimate the total water mass in the envelope to be $10^{-3} M_{\odot}$ and that $93 \%$ of this mass resides in the inner parts, to be compared with $2 \times 10^{-4} M_{\odot}$ and $69 \%$ in the case of a nonthermal contribution.

\subsection{Kinematics and geometry of the region}

The previous section suggests that the $\mathrm{THz}$ water feature (1) is consistent with thermal excitation (even if a nonthermal contribution is possible), while feature (2) could be masing. Different physical conditions, that is, a different spatial origin, could explain these different behaviors. According to Gray et al. (2016), this could for instance be explained by a higher dust temperature in the region where the $-57 \mathrm{~km} \mathrm{~s}^{-1}$ water is excited compared to the $-48 \mathrm{~km} \mathrm{~s}^{-1}$ region (the $\mathrm{o}-\mathrm{H}_{2} \mathrm{O} 8_{2,7}-7_{3,4}$ inversion is lost with increasing dust temperature).

When we plot the $6.7 \mathrm{GHz}$ methanol maser spots and the location of high-mass YSO IRS1a and b from Moscadelli \& Goddi (2014) over our $22 \mathrm{GHz}$ e-MERLIN map, the $-48 \mathrm{~km} \mathrm{~s}^{-1} 22 \mathrm{GHz}$ maser spots appear located close to the IRS1b source, while the $-57 \mathrm{~km} \mathrm{~s}^{-1}$ spots are associated with the IRS1a source. Interestingly, Moscadelli \& Goddi (2014), based on $\mathrm{NH}_{3}$ maps, explain that the gas surrounding IRS1b (a less evolved source) has a lower temperature than the gas observed toward IRS1a, which is a more massive and evolved YSO, with $T$ lower than $250 \mathrm{~K}$. Hence, the higher temperature in IRS1 a might be less suitable for water maser emission at $1296 \mathrm{GHz}$. It is more likely, however, that IRS1a could collisionally quench the maser if the number density is too high. We estimate the critical density (at $T=50 \mathrm{~K}$ ) corresponding to the $1296 \mathrm{GHz}$ transition to be $5 \times 10^{7} \mathrm{~cm}^{-3}$. The gas in the core of IRS1a is thus so dense (number densities of up to a few $10^{8} \mathrm{~cm}^{-3}$ can be reached in the inner part, see Sect. 4.2.1) that it begins to quench the maser action.

The maser spot distribution as derived from our e-MERLIN map provides new information when compared to previous published works. Figure 5 shows the spatial location of the $22 \mathrm{GHz}$ maser features and the $6.7 \mathrm{GHz}$ methanol masers of Moscadelli \& Goddi (2014). According to these authors, the two individual high-mass YSOs, IRS1a and $b$, lie at the center of a line of methanol masers tracing disks with position angles of $\mathrm{PA}=+71^{\circ}$ and $-73^{\circ}$, respectively. The strongest "blue" (i.e., $v \leq-56 \mathrm{~km} \mathrm{~s}^{-1}$ ) maser spots we detect can be associated with IRS1a, others are distributed roughly along a line with PA = $-25^{\circ}$ and another with PA $=-50^{\circ}$. Some similar linear distribution with PA $=-52^{\circ}$ was seen by Surcis et al. (2011), who suggested that these water masers were almost aligned with the $\mathrm{CO}$ NW-SE outflow, elongated $0.3 \mathrm{pc}$ from IRS1a with PA $=-40^{\circ}$ (Kameya et al. 1990; Gaume et al. 1995; Qiu et al. 2011). They proposed that the water masers are pumped by a shock caused by the interaction of the outflow with the infalling gas (observed at scales $\geq 1000 \mathrm{AU}$, Beuther et al. 2013). The e-MERLIN map shows water masers following this NW-SE axis of the CO outflow, but at slower velocities compared to what Qiu et al. (2011) observed in $\mathrm{CO}\left(-78 ;-64 \mathrm{~km} \mathrm{~s}^{-1}\right)$. These $\mathrm{H}_{2} \mathrm{O}$ maser spots might either trace the cavity of the outflow, that is, a cone with an opening angle of $\sim 25^{\circ}$ and $\mathrm{PA} \simeq-40^{\circ}$, or trace two different outflows originating from IRS1a, the outflow at PA $=-25^{\circ}$ being almost perpendicular to the disk.

Maser spots whose velocity is close to the $\mathrm{THz}$ feature (2) (i.e., $-44 ;-50 \mathrm{~km} \mathrm{~s}^{-1}$ ) are located NW from IRS1a and W from IRS1b. They are also distributed along a line with PA $\simeq$ $+28^{\circ}$, that is, NE-SW, which can be associated with the outflow 
observed by Beuther et al. (2013) in $\mathrm{HCO}^{+}(4-3)$ with $\mathrm{PA} \simeq 40^{\circ}$. Moreover, the $\mathrm{NH}_{3}$ (Moscadelli \& Goddi 2014), OCS, $\mathrm{CH}_{3} \mathrm{CN}$, and ${ }^{13} \mathrm{CO}$ (Zhu et al. 2013) observations show a velocity gradient in the same direction ( $\mathrm{PA} \sim 30-40^{\circ}$ ) with line emission at velocities similar to our feature (2) emission in this NE region. Moscadelli \& Goddi (2014) explain these "redshifted" $\mathrm{NH}_{3}$ features toward the NE by an outflow driven by IRS1b that is collimated by its rotating disk. Hence, as proposed above on the basis of a too high temperature toward IRS1a, we propose that the $\mathrm{THz}$ feature (2) is a maser, not associated with IRS1a, and is pumped by shocks that are driven by the IRS1b outflow.

\section{Conclusions}

SOFIA observations toward NGC 7538-IRS1 of the $\mathrm{o}-\mathrm{H}_{2} \mathrm{O}$ $8_{2,7}-7_{3,4}$ line emission were presented. Two separate velocity features were detected: one associated with the source velocity $\left(-57.7 \mathrm{~km} \mathrm{~s}^{-1}\right)$, and another one lies at $-48.4 \mathrm{~km} \mathrm{~s}^{-1}$. By combining these observations with near-simultaneous observations of the $6_{1,6}-5_{2,3}$ masing transition of ortho- $\mathrm{H}_{2} \mathrm{O}$ at $22 \mathrm{GHz}$ with the Effelsberg telescope and with the e-MERLIN interferometer, we discussed the nature of these $\mathrm{THz}$ emission features.

A thermal water model based on HIFI observations can reproduce the $8_{2,7}-7_{3,4}$ line component at the source velocity if the water inner abundance is increased by more than an order of magnitude to $5.2 \times 10^{-5}$ compared to what is estimated by the "HIFI alone" model. In addition, the observed brightness ratio $(1296 / 22)$ for both features was compared to the maser predictions and led us to conclude that while the $\mathrm{THz}$ emission feature at the systemic velocity is mostly thermal, the $-48.4 \mathrm{~km} \mathrm{~s}^{-1}$ feature is likely masing.

We argue that the two line components do not arise from the same location, meaning that different physical conditions could explain these different natures. The thermal emission is excited in the innermost part of the IRS1a protostellar massive object and is an excellent probe of the water reservoir in the inner part of the hot core. We suggest that the maser emission is associated with shocks driven by the IRS1b outflow.

Acknowledgements. We would like to thank Anne Lähteenmäki (Metsähovi Radio Observatory, Aalto University, Finland) for the flux measurements of 3C 84 and Alex Kraus (MPIfR-Bonn, Germany) for having performed and reduced the Effelsberg observations. The Effelsberg 100-m telescope is a facility of the MPIfR (Max-Planck-Institut für Radioastronomie) in Bonn. e-MERLIN is a national facility operated by The University of Manchester on behalf of the Science and Technology Facilities Council (STFC). We thank the SOFIA operations and the GREAT instrument teams, whose support has been essential for the GREAT accomplishments, and the DSI telescope engineering team. Based [in part] on observations made with the NASA/DLR Stratospheric $\mathrm{Ob}$ servatory for Infrared Astronomy. Sofia Science Mission operations are conducted jointly by the Universitis Space Research Association, Inc., under NASA contract NAS297001, and the Deutsches SOFIA Institut, under DLR contract 50 OK 0901. GREAT is a development by the MPI für Radioastronomie and the KOSMA/Universität zu Köln, in cooperation with the MPI für Sonnensystemforschung and the DLR Institut für Planetenforschung. Herschel is an ESA space observatory with science instruments provided by European-led Principal
Investigator consortia and with important participation from NASA. HIFI has been designed and built by a consortium of institutes and university departments from across Europe, Canada and the United States under the leadership of SRON Netherlands Institute for Space Research, Groningen, The Netherlands and with major contributions from Germany, France and the US. Consortium members are: Canada: CSA, U.Waterloo; France: CESR, LAB, LERMA, IRAM; Germany: KOSMA, MPIfR, MPS; Ireland, NUI Maynooth; Italy: ASI, IFSIINAF, Osservatorio Astrofisico di Arcetri- INAF; Netherlands: SRON, TUD; Poland: CAMK, CBK; Spain: Observatorio Astronómico Nacional (IGN), Centro de Astrobiología (CSIC-INTA). Sweden: Chalmers University of Technol ogy - MC2, RSS \& GARD; Onsala Space Observatory; Swedish National Space Board, Stockholm University - Stockholm Observatory; Switzerland: ETH Zurich, FHNW; USA: Caltech, JPL, NHSC.).

\section{References}

Aikawa, Y., Wakelam, V., Garrod, R. T., \& Herbst, E. 2008, ApJ, 674, 984 Beuther, H., Leurini, S., Schilke, P., et al. 2007, A\&A, 466, 1065 Beuther, H., Linz, H., \& Henning, T. 2013, A\&A, 558, A81 Chavarría, L., Herpin, F., Jacq, T., et al. 2010, A\&A, 521, L37 Csengeri, T., Menten, K. M., Wyrowski, F., et al. 2012, A\&A, 542, L8 Daniel, F., \& Cernicharo, J. 2013, A\&A, 553, A70 de Graauw, T., Helmich, F. P., Phillips, T. G., et al. 2010, A\&A, 518, L6 Elitzur, M., Hollenbach, D. J., \& McKee, C. F. 1992, ApJ, 394, 221 Felli, M., Brand, J., Cesaroni, R., et al. 2007, A\&A, 476, 373

Fraser, H. J., Collings, M. P., McCoustra, M. R. S., \& Williams, D. A. 2001, MNRAS, 327, 1165

Galván-Madrid, R., Montes, G., Ramírez, E. A., et al. 2010, ApJ, 713, 423

Gaume, R. A., Goss, W. M., Dickel, H. R., Wilson, T. L., \& Johnston, K. J. 1995 ApJ, 438, 776

Gray, M. D., Baudry, A., Richards, A. M. S., et al. 2016, MNRAS, 456, 374

Herpin, F., Chavarría, L., van der Tak, F., et al. 2012, A\&A, 542, A76

Herpin, F., Chavarría, L., Jacq, T., et al. 2016, A\&A, 587, A139

Heyminck, S., Graf, U. U., Güsten, R., et al. 2012, A\&A, 542, L1

Hogerheijde, M. R., \& van der Tak, F. F. S. 2000, A\&A, 362, 697

Kameya, O., Morita, K.-I., Kawabe, R., \& Ishiguro, M. 1990, ApJ, 355, 562

Karska, A., Herpin, F., Bruderer, S., et al. 2014, A\&A, 562, A45

Klein, B., Hochgürtel, S., Krämer, I., et al. 2012, A\&A, 542, L3

König, C., Urquhart, J. S., Csengeri, T., et al. 2017, A\&A, 599, A139

Minier, V., Booth, R. S., \& Conway, J. E. 2000, A\&A, 362, 1093

Moscadelli, L., \& Goddi, C. 2014, A\&A, 566, A150

Moscadelli, L., Reid, M. J., Menten, K. M., et al. 2009, ApJ, 693, 406

Neufeld, D. A., \& Melnick, G. J. 1991, ApJ, 368, 215

Neufeld, D. A., Melnick, G. J., Kaufman, M. J., et al. 2017, ApJ, 843, 94 Ott, S. 2010, in Astronomical Data Analysis Software and Systems XIX, eds. Y. Mizumoto, K.-I. Morita, \& M. Ohishi, ASP Conf. Ser., 434, 139

Qiu, K., Zhang, Q., \& Menten, K. M. 2011, ApJ, 728, 6

Roelfsema, P. R., Helmich, F. P., Teyssier, D., et al. 2012, A\&A, 537, A17

Sandell, G., Goss, W. M., Wright, M., \& Corder, S. 2009, ApJ, 699, L31

Surcis, G., Vlemmings, W. H. T., Torres, R. M., van Langevelde, H. J., \& Hutawarakorn, K., B. 2011, A\&A, 533, A47

Thomas, H. S., \& Fuller, G. A. 2008, A\&A, 479, 751

van der Tak, F. F. S., van Dishoeck, E. F., Evans, II, N. J., \& Blake, G. A. 2000 ApJ, 537, 283

van der Tak, F. F. S., Walmsley, C. M., Herpin, F., \& Ceccarelli, C. 2006, A\&A, 447, 1011

van der Tak, F. F. S., Chavarría, L., Herpin, F., et al. 2013, A\&A, 554, A83

van Dishoeck, E. F., Kristensen, L. E., Benz, A. O., et al. 2011, PASP, 123, 138

Werner, M. W., Becklin, E. E., Gatley, I., et al. 1979, MNRAS, 188, 463

Wilson, T. L., \& Rood, R. 1994, ARA\&A, 32, 191

Yates, J. A., Field, D., \& Gray, M. D. 1997, MNRAS, 285, 303

Young, E. T., Becklin, E. E., Marcum, P. M., et al. 2012, ApJ, 749, L17

Zhu, L., Zhao, J.-H., Wright, M. C. H., et al. 2013, ApJ, 779, 51 
F. Herpin et al.: First detection of a THz water maser in NGC 7538-IRS1 with SOFIA and new 22 GHz e-MERLIN maps

\section{Appendix A: Water line transitions observed with Herschel/HIFI}

Table A.1. Water line transitions observed with Herschel/HIFI.

\begin{tabular}{|c|c|c|c|c|c|c|c|c|c|}
\hline Water species & $\begin{array}{c}\text { Frequency } \\
{[\mathrm{GHz}]}\end{array}$ & $\begin{array}{l}\text { Wavelength } \\
\qquad[\mu \mathrm{m}]\end{array}$ & $\begin{array}{c}E_{u} \\
{[\mathrm{~K}]}\end{array}$ & $\begin{array}{l}\text { HIFI } \\
\text { band }\end{array}$ & $\begin{array}{c}\text { Beam } \\
{\left[{ }^{\prime \prime}\right]}\end{array}$ & $\eta_{\mathrm{mb}}$ & $\begin{array}{l}T_{\text {sys }} \\
{[\mathrm{K}]}\end{array}$ & $\begin{array}{c}\mathrm{rms} \\
{[\mathrm{mK}]}\end{array}$ & Obsid \\
\hline $\mathrm{o}-\mathrm{H}_{2}^{18} \mathrm{O} 1_{10}-1_{01}^{a}$ & 547.6764 & 547.4 & 60.5 & $1 \mathrm{a}$ & 37.8 & 0.62 & 80 & 58 & 1342202036 \\
\hline $\mathrm{o}-\mathrm{H}_{2}^{17} \mathrm{O} 1_{10}-1_{01}$ & 552.0209 & 543.1 & 61.0 & $1 \mathrm{a}$ & 37.8 & 0.62 & 70 & 42 & $1342198332-3$ \\
\hline $\mathrm{p}-\mathrm{H}_{2}^{18} \mathrm{O} 2_{02}-1_{11}$ & 994.6751 & 301.4 & 100.6 & $4 \mathrm{a}$ & 21.1 & 0.63 & 290 & 69 & $1342197964-5$ \\
\hline $\mathrm{o}-\mathrm{H}_{2}^{18} \mathrm{O} 3_{12}-3_{03}$ & 1095.6274 & 273.8 & 248.7 & $4 b$ & 19.9 & 0.63 & 380 & 48 & $1342200760-1$ \\
\hline $\mathrm{p}-\mathrm{H}_{2}^{18} \mathrm{O} 1_{11}-0_{00}$ & 1101.6982 & 272.1 & 52.9 & $4 \mathrm{~b}$ & 19.9 & 0.63 & 390 & 36 & $1342191663-4,1342197976$ \\
\hline $\mathrm{p}-\mathrm{H}_{2}^{17} \mathrm{O} 1_{11}-0_{00}$ & 1107.1669 & 272.1 & 52.9 & $4 \mathrm{~b}$ & 19.9 & 0.63 & 380 & 37 & $1342200760-1$ \\
\hline $\mathrm{o}-\mathrm{H}_{2}^{17} \mathrm{O} 2_{12}-1_{01}$ & 1662.4644 & 180.3 & 113.6 & $6 b$ & 12.7 & 0.58 & 1410 & 232 & 1342200758 \\
\hline $\mathrm{o}-\mathrm{H}_{2} \mathrm{O} 1_{10}-1_{01}{ }^{a}$ & 556.9361 & 538.3 & 61.0 & 1a & 37.1 & 0.62 & 80 & 65 & 1342202036 \\
\hline $\mathrm{p}-\mathrm{H}_{2} \mathrm{O} 2_{11}-2_{02}$ & 752.0332 & 398.6 & 136.9 & $2 b$ & 28.0 & 0.64 & 90 & 67 & $1342201546-7$ \\
\hline $\mathrm{p}-\mathrm{H}_{2} \mathrm{O} 5_{24}-4_{31} b$ & 970.3150 & 309.0 & 598.8 & $4 \mathrm{a}$ & 21.8 & 0.63 & 620 & 59 & 1342227536 \\
\hline $\mathrm{p}-\mathrm{H}_{2} \mathrm{O} 2_{02}-1_{11}$ & 987.9268 & 303.5 & 100.8 & $4 a$ & 21.3 & 0.63 & 340 & 69 & $1342197964-5$ \\
\hline $\mathrm{o}-\mathrm{H}_{2} \mathrm{O} 3_{12}-3_{03}$ & 1097.3651 & 273.2 & 249.4 & $4 \mathrm{~b}$ & 19.9 & 0.63 & 380 & 49 & $1342200760-1$ \\
\hline $\mathrm{p}-\mathrm{H}_{2} \mathrm{O} 1_{11}-0_{00}$ & 1113.3430 & 269.0 & 53.4 & $4 \mathrm{~b}$ & 19.7 & 0.63 & 395 & 36 & $1342191663-4,1342197976$ \\
\hline $\mathrm{o}-\mathrm{H}_{2} \mathrm{O} 2_{21}-2_{12}$ & 1661.0076 & 180.5 & 194.1 & $6 b$ & 12.7 & 0.58 & 1410 & 232 & 1342200758 \\
\hline $\mathrm{o}-\mathrm{H}_{2} \mathrm{O} 2_{12}-1_{01}$ & 1669.9048 & 179.5 & 114.4 & $6 b$ & 12.6 & 0.58 & 1410 & 232 & 1342200758 \\
\hline
\end{tabular}

Notes. The rms is the noise in $\delta v=1.1 \mathrm{MHz} .{ }^{(a)}$ This line was mapped in OTF mode. ${ }^{(b)}$ Line affected by strong baseline and ripple problems. 


\section{Appendix B: Water maser positions}

Table B.1. Water maser positions and velocities at $22 \mathrm{GHz}$ (e-MERLIN observations of April 2016).

\begin{tabular}{|c|c|c|c|c|c|c|}
\hline IRS & Group & $\begin{array}{c}\text { offset } \mathrm{X}^{a} \\
{\left[{ }^{\prime \prime}\right]}\end{array}$ & $\begin{array}{c}\text { Offset } \mathrm{Y}^{a} \\
{\left[{ }^{\prime \prime}\right]}\end{array}$ & $\begin{array}{c}\text { Peak } S_{v} \\
{[\mathrm{Jy}]}\end{array}$ & $\begin{array}{c}\text { Peak } V_{\mathrm{LSR}} \\
{\left[\mathrm{km} \mathrm{s}^{-1}\right]}\end{array}$ & $\begin{array}{c}\text { Velocity range } \\
{\left[\mathrm{km} \mathrm{s}^{-1}\right]}\end{array}$ \\
\hline 1 & 1 & 0.132 & -0.010 & 17.31 & -45.06 & -44.64 to -45.70 \\
\hline 1 & 2 & 0.133 & 0.005 & 143.23 & -45.27 & -44.43 to -45.91 \\
\hline 1 & 3 & 0.070 & -0.106 & 13.12 & -48.33 & -48.22 to -48.65 \\
\hline 1 & 4 & 0.0762 & -0.095 & 145.27 & -48.12 & -47.49 to -48.96 \\
\hline 1 & 5 & 0.114 & -0.081 & 4.21 & -48.12 & -48.12 to -48.54 \\
\hline 1 & 6 & 0.114 & -0.061 & 4.61 & -48.22 & -48.12 to -48.64 \\
\hline 1 & 7 & 0.123 & -0.060 & 1.01 & -48.12 & -48.12 to -48.33 \\
\hline 1 & 8 & 0.079 & -0.070 & 2.91 & -48.33 & -48.33 to -48.75 \\
\hline 1 & 9 & -0.001 & -0.122 & 9.16 & -54.96 & -54.44 to -55.49 \\
\hline 1 & 10 & -0.042 & -0.160 & 23.23 & -57.91 & -56.54 to -57.91 \\
\hline 1 & 11 & -0.288 & -0.010 & 29.24 & -58.23 & -57.81 to -58.55 \\
\hline 1 & 12 & -0.287 & -0.012 & 62.02 & -58.34 & -57.81 to -59.07 \\
\hline 1 & 13 & -0.278 & -0.037 & 2.92 & -58.12 & -58.12 to -58.34 \\
\hline 1 & 14 & -0.304 & -0.051 & 1.44 & -58.34 & -58.34 to -58.65 \\
\hline 1 & 15 & -0.251 & -0.027 & 0.86 & -58.65 & -58.44 to -58.65 \\
\hline 1 & 16 & -0.238 & 0.012 & 1.88 & -58.44 & -58.44 to -58.65 \\
\hline 1 & 17 & 0.094 & 0.038 & 8.59 & -59.28 & -58.86 to -59.49 \\
\hline 1 & 18 & 0.005 & -0.250 & 112.31 & -60.13 & -59.92 to -60.65 \\
\hline 1 & 19 & 0.006 & -0.234 & 29.61 & -60.23 & -60.13 to -60.44 \\
\hline 1 & 20 & -0.012 & -0.258 & 26.90 & -60.55 & -60.13 to -60.55 \\
\hline 1 & 21 & 0.004 & -0.250 & 964.41 & -60.01 & -59.18 to -61.07 \\
\hline 1 & 22 & 0.020 & -0.2820 & 10.34 & -59.81 & -59.39 to -59.81 \\
\hline 1 & 23 & -0.018 & -0.195 & 12.34 & -59.70 & -59.39 to -59.81 \\
\hline 1 & 24 & 0.036 & -0.319 & 6.97 & -59.70 & -59.39 to -59.81 \\
\hline 1 & 25 & 0.048 & -0.243 & 6.46 & -60.76 & -60.55 to -61.07 \\
\hline 1 & 26 & -0.111 & -0.154 & 27.73 & -61.39 & -60.86 to -62.23 \\
\hline $1 \mathrm{~S}$ & 1 & -0.163 & -1.201 & 14.32 & -70.66 & -69.20 to -70.98 \\
\hline $1 \mathrm{~S}$ & 2 & -0.170 & -1.211 & 27.09 & -46.43 & -45.80 to -47.07 \\
\hline 3 & 1 & -5.647 & 2.456 & 14.40 & -56.86 & -56.33 to -57.60 \\
\hline 3 & 2 & -5.642 & 2.474 & 88.55 & -56.76 & -56.12 to -57.91 \\
\hline 9 & 1 & 117.433 & -50.752 & 2.06 & -69.18 & -68.97 to -69.19 \\
\hline 9 & 2 & 117.432 & -50.751 & 2.76 & -69.39 & -69.39 to -69.61 \\
\hline 9 & 3 & 117.436 & -50.742 & 39.13 & -73.61 & -72.45 to -74.98 \\
\hline 11 & 1 & -2.747 & -80.919 & 7.56 & -44.96 & -44.75 to -45.70 \\
\hline 11 & 2 & -2.736 & -80.885 & 32.20 & -45.48 & -45.27 to -45.60 \\
\hline 11 & 3 & -2.743 & -80.926 & 29.41 & -47.06 & -45.91 to -47.80 \\
\hline 11 & 4 & -2.733 & -80.888 & 12.24 & -46.75 & -46.33 to -47.60 \\
\hline 11 & 5 & -2.748 & -80.970 & 2.38 & -47.28 & -46.85 to -47.49 \\
\hline 11 & 6 & -2.738 & -80.984 & 6.84 & -47.06 & -46.64 to -47.59 \\
\hline 11 & 7 & -2.730 & -80.846 & 2.83 & -46.85 & -46.64 to -47.17 \\
\hline 11 & 8 & -2.715 & -80.960 & 1.82 & -46.85 & -46.65 to -47.06 \\
\hline 11 & 9 & -2.684 & -80.908 & 2.05 & -47.38 & -47.17 to -47.38 \\
\hline 11 & 10 & -4.127 & -79.095 & 9.53 & -50.01 & -49.59 to -50.33 \\
\hline 11 & 11 & -4.112 & -79.057 & 5.23 & -49.59 & -49.59 to -50.22 \\
\hline 11 & 12 & -4.116 & -79.160 & 3.88 & -50.12 & -49.91 to -50.22 \\
\hline 11 & 13 & -4.123 & -79.126 & 1.69 & -50.44 & -50.22 to -50.44 \\
\hline 11 & 14 & -4.300 & -80.046 & 3.94 & -53.49 & -53.28 to -53.81 \\
\hline 11 & 15 & -4.293 & -80.012 & 1.64 & -53.81 & -53.38 to -53.81 \\
\hline 11 & 16 & -2.928 & -81.067 & 21.02 & -54.12 & -53.81 to -54.65 \\
\hline 11 & 17 & -2.918 & -81.120 & 11.80 & $-54.44-$ & 53.91 to -54.65 \\
\hline 11 & 18 & -2.931 & -80.992 & 5.31 & -54.23 & -53.91 to -54.44 \\
\hline 11 & 19 & -2.919 & -81.039 & 3.24 & -54.23 & -53.91 to -54.44 \\
\hline 11 & 20 & -2.874 & -81.056 & 2.21 & -54.33 & -54.33 to -54.54 \\
\hline 11 & 21 & -2.793 & -80.885 & 4.52 & -55.60 & -55.07 to -55.70 \\
\hline
\end{tabular}

Notes. ${ }^{(a)}$ All offsets are relative to the position RA $=23 \mathrm{~h} 13 \mathrm{~m} 45.364 \mathrm{~s}$, Dec $\left.=+61^{\circ} 28^{\prime} 10.550^{\prime \prime}\right)$. The uncertainty is smaller than $0.003^{\prime \prime}$. 
Table B.1. continued.

\begin{tabular}{|c|c|c|c|c|c|c|}
\hline IRS & Group & $\begin{array}{c}\text { offset } \mathrm{X}^{a} \\
{\left[{ }^{\prime \prime}\right]} \\
\end{array}$ & $\begin{array}{c}\text { offset } \mathrm{Y}^{a} \\
\left.{ }^{\prime \prime}\right] \\
\end{array}$ & $\begin{array}{c}\text { Peak } S_{v} \\
{[\mathrm{Jy}]} \\
\end{array}$ & $\begin{array}{c}\text { Peak } V_{\text {LSR }} \\
{\left[\mathrm{km} \mathrm{s}^{-1}\right]} \\
\end{array}$ & $\begin{array}{c}\text { Velocity range } \\
{\left[\mathrm{km} \mathrm{s}^{-1}\right]} \\
\end{array}$ \\
\hline 11 & 22 & -2.784 & -80.861 & 2.92 & -55.28 & -55.07 to -55.49 \\
\hline 11 & 23 & -2.914 & -81.168 & 1.77 & -55.81 & -55.81 to -56.02 \\
\hline 11 & 24 & -1.121 & -81.967 & 10.40 & -56.02 & -55.91 to -57.97 \\
\hline 11 & 25 & -1.112 & -81.940 & 1.90 & -56.23 & -56.23 to -56.65 \\
\hline 11 & 26 & -4.594 & -79.578 & 10.02 & -57.07 & -56.22 to -57.18 \\
\hline 11 & 27 & -2.797 & -80.876 & 19.98 & -57.07 & -56.33 to -57.49 \\
\hline 11 & 28 & -2.871 & -81.223 & 2.77 & -56.44 & -56.44 to -56.97 \\
\hline 11 & 29 & -2.882 & -81.287 & 3.08 & -56.97 & -56.97 to -57.39 \\
\hline 11 & 30 & -2.879 & -81.252 & 20.05 & -56.97 & -56.44 to -57.49 \\
\hline 11 & 31 & -2.839 & -81.272 & 4.61 & -56.86 & -56.54 to -56.97 \\
\hline 11 & 32 & -2.787 & -80.850 & 7.56 & -57.18 & -56.65 to -57.39 \\
\hline 11 & 33 & -2.761 & -80.925 & 3.04 & -56.97 & -56.65 to -56.97 \\
\hline 11 & 34 & -2.970 & -80.858 & 11.43 & -57.81 & -56.97 to -57.81 \\
\hline 11 & 35 & -2.763 & -80.905 & 3.31 & -57.07 & -57.07 to -57.28 \\
\hline 11 & 36 & -2.789 & -80.938 & 2.40 & -57.28 & -57.07 to -57.28 \\
\hline 11 & 37 & -2.840 & -81.266 & 2.72 & -57.07 & -57.07 to -57.28 \\
\hline 11 & 38 & -2.952 & -80.824 & 3.21 & -57.28 & -57.28 to -57.70 \\
\hline 11 & 39 & -4.240 & -79.974 & 81.11 & -58.02 & -57.49 to -58.76 \\
\hline 11 & 40 & -4.212 & -80.030 & 38.97 & -57.91 & -57.60 to -58.23 \\
\hline 11 & 41 & -4.222 & -79.902 & 22.69 & -58.02 & -57.60 to -58.55 \\
\hline 11 & 42 & -4.227 & -79.945 & 36.30 & -58.02 & -57.70 to -58.55 \\
\hline 11 & 43 & -4.173 & -79.965 & 22.74 & -58.23 & -57.81 to -58.44 \\
\hline 11 & 44 & -4.233 & -80.036 & 13.97 & -58.23 & -58.12 to -58.65 \\
\hline 11 & 45 & -4.242 & -80.017 & 34.93 & -58.34 & -57.91 to -58.76 \\
\hline 11 & 46 & -4.303 & -79.883 & 15.34 & -58.02 & -57.91 to -58.34 \\
\hline 11 & 47 & -3.068 & -80.774 & 28.26 & -60.23 & -59.92 to -60.76 \\
\hline 11 & 48 & -3.055 & -80.732 & 15.82 & -60.34 & -60.13 to -60.44 \\
\hline 11 & 49 & -3.038 & -80.696 & 9.06 & -60.34 & -60.34 to -60.55 \\
\hline 11 & 50 & -3.123 & -80.708 & 5.96 & -61.81 & -61.49 to -62.02 \\
\hline 11 & 51 & -3.115 & -80.665 & 2.34 & -61.71 & -61.60 to -61.92 \\
\hline 11 & 52 & -3.172 & -80.408 & 1.77 & -62.23 & -62.23 to -62.55 \\
\hline 11 & 53 & -3.162 & -80.369 & 2.63 & -62.23 & -62.23 to -62.55 \\
\hline 11 & 54 & -2.800 & -80.898 & 50.61 & -63.81 & -63.71 to -64.34 \\
\hline 11 & 55 & -2.802 & -80.879 & 245.82 & -63.39 & -62.44 to -64.44 \\
\hline 11 & 56 & -2.791 & -80.843 & 221.66 & -63.18 & -62.44 to -65.08 \\
\hline 11 & 57 & -2.767 & -80.918 & 114.46 & -63.18 & -62.65 to -63.19 \\
\hline 11 & 58 & -2.826 & -80.779 & 22.41 & -63.18 & -62.76 to -63.29 \\
\hline 11 & 59 & -2.783 & -80.800 & 54.47 & -63.39 & -62.86 to -63.71 \\
\hline 11 & 60 & -2.791 & -80.934 & 159.10 & -63.30 & -62.76 to -64.13 \\
\hline 11 & 61 & -2.877 & -80.791 & 107.47 & -63.60 & -62.86 to -64.02 \\
\hline 11 & 62 & -2.734 & -80.850 & 3.92 & -63.30 & -63.39 to -63.60 \\
\hline 11 & 63 & -2.734 & -80.857 & 128.98 & -63.39 & -62.97 to -64.02 \\
\hline 11 & 64 & -3.175 & -80.372 & 50.63 & -63.50 & -63.39 to -64.87 \\
\hline 11 & 65 & -3.177 & -80.414 & 25.18 & -64.04 & -63.60 to -64.55 \\
\hline 11 & 66 & -2.775 & -80.799 & 3.42 & -63.92 & -63.92 to -64.13 \\
\hline 11 & 67 & -3.165 & -80.336 & 5.61 & -64.34 & -64.13 to -64.76 \\
\hline 11 & 68 & -2.778 & -80.815 & 2.26 & -64.34 & -64.23 to -64.66 \\
\hline New & 1 & -11.957 & -21.192 & 19.79 & -46.12 & -45.17 to -47.06 \\
\hline New & 2 & -11.960 & -21.199 & 3.81 & -52.96 & -52.75 to -53.28 \\
\hline New & 3 & -11.980 & -21.215 & 8.31 & -56.12 & -55.49 to -56.86 \\
\hline New & 4 & -13.992 & -23.778 & 28.29 & -57.81 & -57.07 to -58.02 \\
\hline New & 5 & -13.989 & -23.756 & 198.18 & -57.60 & -56.33 to -59.49 \\
\hline New & 6 & -13.964 & -23.785 & 2.68 & -56.97 & -56.65 to -56.97 \\
\hline New & 7 & -11.997 & -21.222 & 35.84 & -63.39 & -61.39 to -63.39 \\
\hline
\end{tabular}

